\title{
Comparison principle for second order elliptic operators and applications
}

\section{Principe de comparaison pour opérateurs elliptiques du second ordre et applications}

\author{
Rabah Tahraoui a,b

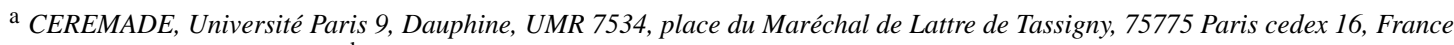 \\ b I.U.F.M. Rouen 2, rue du Tronquet, 76131 Mont-St-Aignan, France
}

Received 21 November 2003; received in revised form 14 January 2005; accepted 16 February 2005

Available online 20 June 2005

\section{Abstract}

Given two elliptic operators $A_{1}$ and $A_{2}$, let us consider the following problems:

$$
\begin{cases}-A_{i} u_{i}=f_{i}, & f_{i} \geqslant 0 \text { in } \Omega, \\ \left.u_{i}\right|_{\partial_{\Omega}}=0, & i=1,2 .\end{cases}
$$

In this work, we give some sufficient conditions ensuring the following comparison principle:

$$
u_{1}(x) \geqslant u_{2}(x) \quad \forall x \in \Omega .
$$

As application, we study the behaviour, as $|x| \rightarrow+\infty$, of the solution $u$ of the problem

$$
\left\{\begin{array}{l}
-A u=f \quad \text { in } \mathbb{R}^{N}, \\
\lim _{|x| \rightarrow+\infty} u(x)=0
\end{array}\right.
$$

(C) 2006 L'Association Publications de l'Institut Henri Poincaré. Published by Elsevier B.V. All rights reserved

\section{Résumé}

Etant donnés deux opérateurs $A_{1}$ and $A_{2}$, on considère les deux problèmes suivants :

$$
\begin{cases}-A_{i} u_{i}=f_{i}, & f_{i} \geqslant 0 \text { in } \Omega, \\ \left.u_{i}\right|_{\partial_{\Omega}}=0, & i=1,2 .\end{cases}
$$

E-mail address: tahraoui@ ceremade.dauphine.fr (R. Tahraoui). 
Dans ce travail, on se propose de donner des conditions suffisantes portant sur $A_{i}, f_{i}$ permettant de comparer les deux solutions $u_{1}$ et $u_{2}$ :

$$
u_{1}(x) \geqslant u_{2}(x) \quad \forall x \in \Omega .
$$

Comme application, nous étudions le comportement asymptotique, quand $|x| \rightarrow+\infty$, de la solution $u$ du problème suivant :

$$
\left\{\begin{array}{l}
-A u=f \quad \text { in } \mathbb{R}^{N}, \\
\lim _{|x| \rightarrow+\infty} u(x)=0 .
\end{array}\right.
$$

(C) 2006 L'Association Publications de l'Institut Henri Poincaré. Published by Elsevier B.V. All rights reserved

\section{Introduction}

We are concerned with Comparison Principle for linear second order partial differential operators. Comparison Principle, as Maximum Principle, is a mathematical tool which is used frequently in many fields of mathematics [1-10]. In this paper we propose to give a new Comparison Principle. More precisely, let us consider $u_{A}$ and $u_{B}$ solution of

$$
\begin{array}{ll}
-A u_{A}=f_{1}, & u_{A} \in H_{0}^{1}(\Omega), \\
-B u_{B}=f_{2}, & u_{B} \in H_{0}^{1}(\Omega),
\end{array}
$$

where $A=\sum_{i j} a_{i j} \frac{\partial^{2}}{\partial x_{i} \partial x_{j}}$ and $B=\sum_{i j} b_{i j} \frac{\partial^{2}}{\partial x_{i} \partial x_{j}}$ are uniformly elliptic operators. Let us denote by $a$ and $b$, respectively, the matrices $\left(a_{i j}\right)$ and $\left(b_{i j}\right) . \Omega$ is a bounded open subset of $\mathbb{R}^{N}, N \geqslant 3$, and $f_{i}, i=1,2$, are nonnegative, for simplicity, functions belonging to $L^{2}(\Omega)$. We deal with the following natural question: can we compare $u_{A}$ and $u_{B}$ in some sense? We are looking for reasonable assumptions about the link between $\left(A, f_{1}\right)$ and $\left(B, f_{2}\right)$, to imply that the answer to this question is positive, that is to say:

$$
u_{A}(x)>u_{B}(x) \text { a.e. } x \in \Omega \text {. }
$$

Let us give a precise idea of our result in a simple but significant setting: let $\Omega$ be an open ball $B(0, R)$ of $\mathbb{R}^{3}$. Let us assume that $f_{i}(x)=f_{i}(|x|), i=1,2$, and $a=\left(a_{i j}\right)$ and $b=\left(b_{i j}\right)$ are constant matrices. Our result can be claimed as follow: If $\left(A, f_{1}\right)$ and $\left(B, f_{2}\right)$ are related by

$$
\begin{aligned}
& \int_{y}^{R^{2}} \frac{1}{\lambda_{3}(a)}\left[\int_{0}^{t}\left(\frac{s}{t}\right)^{\operatorname{Tr}(a) /\left(2 \lambda_{1}(a)\right)} \frac{f_{1}(s)}{s} \mathrm{~d} s\right] \mathrm{d} t \\
& \geqslant \int_{y}^{R^{2}} \frac{1}{\lambda_{1}(b)}\left[\int_{0}^{t}\left(\frac{s}{t}\right)^{\operatorname{Tr}(b) /\left(2 \lambda_{3}(b)\right)} \frac{f_{2}(s)}{s} \mathrm{~d} s\right] \mathrm{d} t, \quad \forall y \in\left[0, R^{2}\right]
\end{aligned}
$$

then we have

$$
u_{A}(x)>u_{B}(x) \text { a.e. } x \in B(0, R),
$$

where for any matrix $c=\left(c_{i j}\right), \lambda_{1}(c), \lambda_{2}(c)$ and $\lambda_{3}(c)$ stand for, respectively, the first, the second and the third eigenvalue of $c$ and $\operatorname{Tr}(c)=\lambda_{1}(c)+\lambda_{2}(c)+\lambda_{3}(c)$.

Finally let us point out that the assumption $(\mathrm{H})$ is sufficiently sharp: indeed it is possible to give a condition infirming $(\mathrm{H})$ and implying that $(\mathrm{I})$ does not hold. 
We use our results to give the behaviour of the solution of $\left(E_{A}\right)$ in the all space $\mathbb{R}^{N}, N \geqslant 3$ :

$$
\left\{\begin{array}{l}
-A u=f \quad \text { in } \mathbb{R}^{N}, f \geqslant 0 \\
\lim _{|x| \rightarrow+\infty} u(x)=0
\end{array}\right.
$$

We assume that the support of $f(\cdot)$ is compact. The result depends to the geometry of the matrix $a(x)=\left(a_{i j}(x)\right)$. Without any hypothesis about this geometry the more general result is: there exist some positive constants $c_{1}, c_{2}$ and $R$ such that for any $x \in \mathbb{R}^{N},|x| \geqslant R$ we have

$$
\frac{c_{1}}{|x|^{2(\bar{\theta}-1)}} \leqslant u(x) \leqslant \frac{c_{2}}{|x|^{2(\underline{\theta}-1)}}
$$

where

$$
\begin{aligned}
& \bar{\theta}=\lim _{R \rightarrow+\infty} \sup _{|x| \geqslant R} \Lambda(x), \\
& \underline{\theta}=\lim _{R \rightarrow+\infty} \inf _{|x| \geqslant R} \Lambda(x)
\end{aligned}
$$

with

$$
\Lambda(x)=\frac{\operatorname{Tr}(a(x))}{\left(2 /|x|^{2}\right) \sum_{i j} a_{i j}(x) \cdot x_{i} x_{j}} .
$$

In some sense this result is optimal. Indeed in the case $\mathrm{A}$ is the Laplace operator $\bar{\theta}=\underline{\theta}=N / 2$ and thus we obtain the classical optimal result:

$$
u(x) \sim \frac{c}{|x|^{N-2}} \quad \text { as }|x| \text { goes to infinity. }
$$

Finally, let us point out that we can obtain some results more precise than $(*)$ if we use some adequate hypothesis.

\section{Notations, hypothesis and method}

\subsection{Notations and hypothesis}

Let $\Omega$ be a bounded connected open subset of $\mathbb{R}^{N}, N \geqslant 3$. We can study the case $N=2$ similarly. For any uniformly elliptic operator

$$
C=\sum_{i j} c_{i j}(x) \frac{\partial^{2}}{\partial x_{i} \partial x_{j}}, \quad \text { with } c_{i j}=c_{j i} \forall i, j,
$$

we set $c=c(x)$ the matrix of its coefficients. $c$ is a symmetric definite positive matrix. We assume that $c$ is Lipschitz continuous matrix. We denote by

$$
\lambda_{1}(c) \leqslant \lambda_{2}(c) \leqslant \cdots \leqslant \lambda_{N}(c)
$$

the eigenvalues of matrix $c$. Let us point out that $\lambda_{i}(c)$ depends on $x$ in $\Omega$. For any $c^{2}(\bar{\Omega})$-function $\varphi$ we set

$$
\begin{aligned}
& \left|\nabla_{C} \varphi\right|^{2}=\sum_{i j} c_{i j} \frac{\partial \varphi}{\partial x_{i}} \frac{\partial \varphi}{\partial x_{j}}, \\
& \Lambda_{1}(c, \varphi)=\Lambda_{1}(c(x), \varphi(x))=\sup _{\sigma \in G_{N}} \sum_{i} \lambda_{i}(c) \lambda_{\sigma(i)}\left(D^{2} \varphi(x)\right), \quad \forall x \in \bar{\Omega}, \\
& \Lambda_{N}(c, \varphi)=\Lambda_{N}(c(x), \varphi(x))=\inf _{\sigma \in G_{N}} \sum_{i} \lambda_{i}(c) \lambda_{\sigma(i)}\left(D^{2} \varphi(x)\right), \quad \forall x \in \bar{\Omega},
\end{aligned}
$$


where $D^{2} \varphi$ stands for the Hessian matrix of $\varphi$ and $G_{N}$ for the permutation group of the set $\{1,2, \ldots, N\}$.

Let us assume that $\varphi$ is such that the function $\varphi(x) /|\nabla \varphi(x)|^{2}$ is well defined in $\bar{\Omega}$. This holds for instance if $\varphi$ is convex as we will see later.

Let us consider the two following functions:

$$
\Lambda_{1}(c, \varphi) \cdot \frac{\varphi}{\left|\nabla_{c} \varphi\right|^{2}}
$$

and

$$
\Lambda_{N}(c, \varphi) \cdot \frac{\varphi}{\left|\nabla_{c} \varphi\right|^{2}} .
$$

These functions are well defined in $\Omega$ since we have

$$
\frac{1}{\lambda_{N}(c)} \frac{\varphi}{|\nabla \varphi|^{2}} \leqslant \frac{\varphi}{\left|\nabla_{c} \varphi\right|^{2}}=\frac{|\nabla \varphi|^{2}}{\left|\nabla_{c} \varphi\right|^{2}} \cdot \frac{\varphi}{|\nabla \varphi|^{2}} \leqslant \frac{1}{\lambda_{1}(c)} \cdot \frac{\varphi}{|\nabla \varphi|^{2}}
$$

and since $\varphi(x) /|\nabla \varphi(x)|^{2}$ is well defined. Let us define for any $t \in[0, T]$

$$
\begin{aligned}
& Q_{1}^{c, \varphi}(t)=\sup \left\{\Lambda_{1}(c(x), \varphi(x)) \cdot \frac{\varphi(x)}{\left|\nabla_{c} \varphi(x)\right|^{2}} \mid x \in \bar{\Omega}, \varphi(x)=t\right\}, \\
& Q_{N}^{c, \varphi}(t)=\inf \left\{\Lambda_{N}(c(x), \varphi(x)) \cdot \frac{\varphi(x)}{\left|\nabla_{c} \varphi(x)\right|^{2}} \mid x \in \bar{\Omega}, \varphi(x)=t\right\}
\end{aligned}
$$

where $T=\sup \{\varphi(x) \mid x \in \bar{\Omega}\}$. It is clear that $Q_{i}^{c, \varphi}(\cdot)$ is bounded in [0,T]. The function $\varphi$ will be precised later. Its role is crucial to establish spectral estimates from above and below of the function $C \varphi /\left|\nabla_{c} \varphi\right|^{2}$, that we need to state our main result (cf. Propositions 7 and 8 and Theorem 9). Let $f_{1}$ and $f_{2}$ be two non negative functions. Let us set, from any $t \in[0, T]$,

$$
\begin{aligned}
& k_{1}^{c, \varphi}(t)=\inf \left[\frac{f_{1}(x) \cdot \varphi(x)}{\left|\nabla_{c} \varphi(x)\right|^{2}} \mid x \in \bar{\Omega}, \varphi(x)=t\right], \\
& k_{2}^{c, \varphi}(t)=\sup \left[\frac{f_{2}(x) \cdot \varphi(x)}{\left|\nabla_{c} \varphi(x)\right|^{2}} \mid x \in \bar{\Omega}, \varphi(x)=t\right] .
\end{aligned}
$$

Let us point out that $k_{i}^{c, \varphi}, i=1,2$, are well defined since we have

$$
\frac{1}{\lambda_{N}(c)} \cdot \frac{\varphi}{|\nabla \varphi|^{2}} \leqslant \frac{\varphi}{\left|\nabla_{c} \varphi\right|^{2}} \leqslant \frac{\varphi}{|\nabla \varphi|^{2}} \cdot \frac{1}{\lambda_{1}(c)}
$$

and since $\frac{\varphi}{|\nabla \varphi|^{2}}$ is well defined.

\subsection{Method}

The role of the function $\varphi$ is crucial in our work. Its choice depends on the geometry of $\Omega$ and on the form of operator $A$. We call $\varphi$ shape function [7,9-11]. Our idea is simple: to give a positive answer, it suffices to get a subsolution $r_{A}(\cdot)$ of $\left(E_{A}\right)$ and a supersolution $r_{B}(\cdot)$ of $\left(E_{B}\right)$, such that

$$
r_{A}(x) \geqslant r_{B}(x) \text { a.e. } x \in \Omega \text {. }
$$

How to construct these functions? For this we use a shape function $\varphi$ : For instance, we look for $r_{A}(\cdot)$ in the form

$$
r_{A}(\cdot)=r_{a} \circ \varphi(\cdot)
$$

where $r_{a}(\cdot)$ is a suitable function of one variable. What is the link between $r_{a}(\cdot)$ and $u_{A}(\cdot)$ ? The function $r_{a}(\cdot)$ is the solution of an ordinary differential equation constructed from the partial differential equation $\left(E_{A}\right)$. The main 
difficulty is to establish an ordinary differential equation which is as close as possible to $\left(E_{A}\right)$, in some sense. For this we need some sharp spectral estimates that we state in the case $\Omega$ and $\varphi$ convex. The general case will be deduced from the first one by a deformation process.

\section{Preliminary results. Some spectral estimates}

In the sequel we need the following result proved in [3] (if also [1]):

Theorem 1. Let $a=\left(a_{i j}\right)$ and $b=\left(b_{i j}\right)$ be two $n$-square symmetric positive matrices. Let us denote by $\lambda_{i}(a)$, respectively $\lambda_{i}(b)$, the eigenvalues of $a$, respectively of $b, i=1,2, \ldots, N$. Then we have

$$
\min _{\sigma \in G_{N}} \sum_{i=1}^{N} \lambda_{i}(a) \lambda_{\sigma(i)}(b) \leqslant \operatorname{Tr}(a \cdot b) \leqslant \sup _{\sigma \in G_{N}} \sum_{i=1}^{N} \lambda_{i}(a) \lambda_{\sigma(i)}(b)
$$

where $G_{N}$ stands for the permutation group of the set $\{1,2, \ldots, N\}$.

Proposition 1. Let us consider the operator

$$
C=\sum c_{i j} \frac{\partial^{2}}{\partial x_{i} \partial x_{j}}
$$

where $c=\left(c_{i j}\right)$ is a positive symmetric matrix. Let $\varphi$ be a convex function. Then we have $C \varphi \geqslant 0$.

Proposition 2. Let $\Omega$ be an open convex subset of $\mathbb{R}^{N}$ and let $\varphi$ be a regular strictly convex function defined on $\Omega$ and such that:

$$
\begin{aligned}
& \varphi(x)>0 \quad \forall x \in \Omega \backslash\left\{x_{0}\right\}, \\
& \varphi\left(x_{0}\right)=0,\left.\quad \varphi\right|_{\partial \Omega}=T, \quad \text { some positive constant. }
\end{aligned}
$$

Then we have:

$$
0<c_{1}=\inf _{\bar{\Omega}} \frac{\varphi(x)}{|\nabla \varphi(x)|^{2}} \leqslant \frac{\varphi(x)}{|\nabla \varphi(x)|^{2}} \leqslant \sup _{\bar{\Omega}} \frac{\varphi(x)}{|\nabla \varphi(x)|^{2}}=c_{2}<+\infty .
$$

The proof of this result is elementary. We omit it.

Remark. It is not interesting to choose $x_{0}$ close to the boundary of $\Omega$; for instance, a good choice of $x_{0}$ is the so called harmonic center of the convex $\Omega$ [2].

Proposition 3. Let us consider the operator

$$
C=\sum_{j, i=1}^{N} c_{i j}(x) \frac{\partial^{2}}{\partial x_{i} \partial x_{j}}
$$

where $c=\left(c_{i j}(x)\right)_{i j}$ is a positive symmetric matrix. Let $\varphi$ be a strictly convex function satisfying the assumption of Proposition 2. Then we have the following spectral estimates:

$$
\left\{\begin{array}{l}
\frac{Q_{N}^{c, \varphi}(t)}{t} \leqslant \frac{(C \varphi)(x)}{\left|\nabla_{c} \varphi(x)\right|^{2}} \leqslant \frac{Q_{1}^{c, \varphi}(t)}{t}, \\
\forall x \in\{y \in \Omega \mid \varphi(y)=t\}, \quad \forall t \in] 0, T] .
\end{array}\right.
$$


Proof. First of all, let us remark that we have

$$
C \varphi=\operatorname{Tr}\left(c \cdot D^{2} \varphi\right)
$$

where $D^{2} \varphi$ stands for the Hessian matrix of $\varphi$. Following Theorem 1 we have, for any $x \in \bar{\Omega}$,

$$
\min _{\sigma \in G_{N}} \sum_{i} \lambda_{i}(c(x)) \cdot \lambda_{\sigma(i)}\left(D^{2} \varphi(x)\right) \leqslant C \varphi(x) \leqslant \max _{\sigma \in G_{N}} \sum_{i} \lambda_{i}(c(x)) \cdot \lambda_{\sigma(i)}\left(D^{2} \varphi(x)\right) .
$$

From Proposition 2 the functions $Q_{1}^{c, \varphi}(t)$ and $Q_{N}^{c, \varphi}(t)$ given in (5) and (4) are well defined. Thus the result follows by writing

$$
\frac{1}{\left|\nabla_{c} \varphi(x)\right|^{2}}=\frac{\varphi(x)}{\left|\nabla_{c} \varphi(x)\right|^{2}} \cdot \frac{1}{\varphi(x)} \quad \forall x \neq x_{0}
$$

Remark. Let us point out that in the interesting particular case $C$ is the Laplace operator, the previous result becomes:

$$
\left\{\begin{array}{l}
\frac{Q_{N}^{c, \varphi}(t)}{t} \leqslant \frac{\Delta \varphi(x)}{|\nabla \varphi|^{2}} \leqslant \frac{Q_{1}^{c, \varphi}(t)}{t}, \\
\forall x \in\{y \mid \varphi(y)=t\}, \quad \forall t \in] 0, T]
\end{array}\right.
$$

where

$$
\begin{aligned}
& Q_{1}^{c, \varphi}(t)=\sup \left\{\frac{\varphi(x) \Delta \varphi(x)}{|\nabla \varphi(x)|^{2}} \mid x \in \bar{\Omega}, \varphi(x)=t\right\}, \\
& Q_{N}^{c, \varphi}(t)=\inf \left\{\frac{\varphi(x) \Delta \varphi(x)}{|\nabla \varphi(x)|^{2}} \mid x \in \bar{\Omega}, \varphi(x)=t\right\} .
\end{aligned}
$$

And in addition, if $\Omega=B(0, R), \varphi(x)=|x|^{2}$ we obtain explicitly:

$$
\frac{\Delta \varphi(x)}{|\nabla \varphi(x)|^{2}}=\frac{N}{2 \varphi(x)}
$$

and thus we have

$$
Q_{1}^{c, \varphi}(t)=Q_{N}^{c, \varphi}(t)=\frac{N}{2}
$$

Let us point out that in some particular but realistic cases the result of Proposition 3 takes a simpler form, as mentioned in Corollaries 4 and 5.

Corollary 4. Assume that $c=\left(c_{i j}\right)$ does not depends on $x, \Omega=\left\{x \in \mathbb{R}^{N} \mid \varphi(x)<T\right\}$, where $\varphi(x)=\sum_{i=1}^{N} a_{i} x_{i}^{2}$, with $a_{i}>0, \forall i=1, \ldots, N$. Then we have:

$$
\left\{\begin{array}{l}
\frac{\Lambda_{N}(c, \varphi)}{\lambda_{N}(d)} \cdot \frac{\alpha_{N}}{2 t} \leqslant \frac{C \varphi(x)}{\left|\nabla_{c} \varphi(x)\right|^{2}} \leqslant \frac{\Lambda_{1}(c, \varphi)}{\lambda_{1}(d)} \cdot \frac{\alpha_{1}}{2 t}, \\
\forall x \in\{x \in \Omega \mid \varphi(x)=t\}, \quad \forall t \in] 0, T],
\end{array}\right.
$$

where

$$
\Lambda_{1}(c, \varphi)=\sup _{\sigma \in G_{N}} \sum_{i} \lambda_{i}(c) a_{\sigma(i)}, \quad \Lambda_{N}(c, \varphi)=\inf _{\sigma \in G_{N}} \sum_{i} \lambda_{i}(c) a_{\sigma(i)},
$$

where $d=\left(d_{i j}\right)$ is defined by $d_{i j}=c_{i j} \cdot a_{i} \cdot a_{j}$, and

$$
\alpha_{1}=\max _{i} a_{i}, \quad \alpha_{N}=\min _{i} a_{i} .
$$


Corollary 5. Assume that $c=\left(c_{i j}\right)$ does not depend on $x$, and let $\Omega=B(0, R)$ be the open ball of $\mathbb{R}^{N}$ centred at 0 , with radius $R$. Let us set $\varphi(x)=\sum_{i} x_{i}^{2}$. Then we have

$$
\frac{\operatorname{Tr} c}{\lambda_{N}(c)} \cdot \frac{1}{4 t} \leqslant \frac{C \varphi(x)}{\left|\nabla_{c} \varphi(x)\right|^{2}} \leqslant \frac{\operatorname{Tr} c}{\lambda_{1}(c)} \cdot \frac{1}{4 t}
$$

$\left.\forall x \in\{y \in B(0, R) \mid \varphi(y)=t\} \forall t \in] 0, T=R^{2}\right]$.

\section{Ordinary differential equation linked with operators $A$ and $B$ : construction of super and sub solution of $\left(E_{A}\right)$ and $\left(E_{B}\right)$ respectively}

We are going to introduce an ordinary differential equation denoted (E) which is essential to construct the supersolution $r_{1}$ of $\left(E_{B}\right)$ and the subsolution $r_{2}$ of $\left(E_{A}\right): r_{1}$ and $r_{2}$ will be constructed from a solution of (E). Let us set

$$
\left.\left.q(t)=\frac{Q(t)}{t} \quad \text { for any } t \text { belonging to }\right] 0, T\right]
$$

where $Q(t)$ is a positive function defined on $[0, T]$ such that $0<\delta_{1} \leqslant Q(t) \leqslant \delta_{2}$, and which will play the role of $Q_{i}^{c, \varphi}$ in (4) and (5). Let us consider the following ordinary differential equation:

$$
\left\{\begin{array}{l}
\left.\left.-\omega^{\prime \prime}(t)-q(t) \omega^{\prime}(t)=\frac{k(t)}{t} \geqslant 0, \quad \text { in }\right] 0, T\right], \\
\omega^{\prime}(0)=0, \quad \omega(T)=0
\end{array}\right.
$$

where $k$ is some function which will play the role of $k_{i}^{c, \varphi}$ in (6) and (7). We assume that $\int_{0}^{T} \frac{k(s)}{s} \mathrm{~d} s<+\infty$. To write (E) in some canonical way, let us introduce the function $\beta$ solution of

$$
\left\{\begin{array}{l}
\left.\left.\frac{\beta^{\prime}(t)}{\beta(t)}=\frac{Q(t)}{t} \quad \text { in }\right] 0, T\right] \\
\beta(0)=0 .
\end{array}\right.
$$

Let us remark that $\beta$ satisfies:

$$
\left(\frac{s}{t}\right)^{\delta_{2}} \leqslant \frac{\beta(s)}{\beta(t)} \leqslant\left(\frac{s}{t}\right)^{\delta_{1}}
$$

for any $s$ and any $t$ such that $0<s \leqslant t$. This implies

$$
c \cdot s^{\delta_{2}} \leqslant \beta(s) \leqslant c \cdot s^{\delta_{1}}
$$

in some neighbourhood of 0 . Then Eq. (E) can be rewritten as:

$$
\left\{\begin{array}{l}
\left.-\frac{1}{\beta(t)}\left(\beta(t) \omega^{\prime}(t)\right)^{\prime}=\frac{k(t)}{t} \geqslant 0 \quad \text { in }\right] 0, T[ \\
\omega^{\prime}(0)=0, \quad \omega(T)=0 .
\end{array}\right.
$$

It is easy to see that the solution of (9) is

$$
\omega(y)=\int_{y}^{T} \frac{1}{\beta(t)} \int_{0}^{t} \frac{\beta(s) k(s)}{s} \mathrm{~d} s \mathrm{~d} t,
$$


and it satisfies

$$
\left\{\begin{array}{l}
\left.0 \leqslant \omega(t) \leqslant(T-t) \cdot \int_{0}^{T} \frac{k(s)}{s} \mathrm{~d} s \quad \text { in }\right] 0, T[, \\
\left.0 \leqslant-\omega^{\prime}(t) \leqslant \int_{0}^{t} \frac{k(s)}{s} \mathrm{~d} s \quad \text { in }\right] 0, T[.
\end{array}\right.
$$

Thus we have proved the following result

Proposition 6. Function $\omega$ given in (9) and (10) is the unique solution of Eq. (E).

\subsection{Subsolution of equation}

$$
\left\{\begin{array}{l}
-A u_{A}=f_{1} \quad \text { in } \Omega, \quad a=\left(a_{i j}\right), \\
\left.u_{A}\right|_{\partial \Omega}=0 .
\end{array}\right.
$$

To use Proposition 2, let us suppose that $\Omega$ is convex and $\varphi$ is regular in $\bar{\Omega}$ and strictly convex such that:

$$
\begin{array}{ll}
\varphi(x)=T & \forall x \in \partial \Omega, \\
\varphi\left(x_{0}\right)=0 & \text { for some } x_{0} \in \Omega .
\end{array}
$$

In order to simplify the exposition of the results, we assume, in a first step, that the functions $k_{1}^{a, \varphi}$ in (6) and $k_{2}^{b, \varphi}$ in (7) satisfy:

$$
\int_{0}^{T} \frac{k_{1}^{a, \varphi}(t)}{t} \mathrm{~d} t<+\infty, \quad \int_{0}^{T} \frac{k_{2}^{b, \varphi}(t)}{t} \mathrm{~d} t<+\infty .
$$

Remark. We will see later that when (H1) is not satisfied, we can proceed by approximation in a neighbourhood of 0 .

Let us set $q_{a}(t)=Q_{1}^{a, \varphi}(t) / t$ where $Q_{1}^{a, \varphi}$ is defined in (4) and let us consider the solution $r_{a}(\cdot)$ of

$$
\left\{\begin{array}{l}
\left.-r_{a}^{\prime \prime}(t)-q_{a}(t) r_{a}^{\prime}(t)=\frac{k_{1}^{a, \varphi}(t)}{t} \quad \text { in }\right] 0, T[, \\
r_{a}^{\prime}(0)=0, \quad r_{a}(T)=0 .
\end{array}\right.
$$

From Proposition $6, r_{a}(\cdot)$ is given by formula (10) i.e.

$$
r_{a}(t)=\int_{t}^{T} \frac{1}{\beta_{a}(s)} \int_{0}^{s} \frac{\beta_{a}(\theta) k_{1}^{a, \varphi}(\theta)}{\theta} \mathrm{d} \theta \mathrm{d} s
$$

where $\beta_{a}(t)$ is solution of

$$
\left\{\begin{array}{l}
\left.\frac{\beta_{a}^{\prime}(t)}{\beta_{a}(t)}=q_{a}(t) \quad \text { in }\right] 0, T[ \\
\beta_{a}(0)=0
\end{array}\right.
$$


Proposition 7. Let us set

$$
r_{A}(x)=r_{a} \circ \varphi(x)=r_{a}(\varphi(x)) .
$$

Then $r_{A}(\cdot)$ is a subsolution of (12).

Proof. First step. In a first step, assume that $r_{a}(\cdot)$ is regular. In a second step, we will study the non regular case by a regularization procedure. From (13) we have:

$$
-r_{a}^{\prime \prime}(\varphi(x))-q_{a}(\varphi(x)) \cdot r_{a}^{\prime}(\varphi(x))=\frac{k_{1}^{a, \varphi}(\varphi(x))}{\varphi(x)} \quad \forall x \in \Omega \backslash\left\{x_{0}\right\} .
$$

From (6) we have:

$$
\frac{k_{1}^{a, \varphi}(\varphi(x))}{\varphi(x)} \leqslant \frac{f_{1}(x)}{\left|\nabla_{a} \varphi(x)\right|^{2}} \quad \text { a.e. } x \in \Omega,
$$

and from (4)

$$
q_{a}(\varphi(x))=\frac{Q_{1}^{a, \varphi}(\varphi(x))}{\varphi(x)} \geqslant \Lambda_{1}(a(x), \varphi(x)) \cdot \frac{1}{\left|\nabla_{a} \varphi(x)\right|^{2}} .
$$

From (16), applying Proposition 3, we get

$$
q_{a}(\varphi(x)) \geqslant \frac{(A \varphi)(x)}{\left|\nabla_{a} \varphi(x)\right|^{2}} \quad \forall x \in \Omega \backslash\left\{x_{0}\right\} .
$$

Since $r_{a}^{\prime}(t) \leqslant 0$ for any $t$, by (17) we can estimate from below the left-hand side of (14.1) and by (15) we can estimate from above the right-hand side of (14.1). We obtain:

$$
-r_{a}^{\prime \prime}(\varphi(x))+\frac{A \varphi(x)}{\left|\nabla_{a} \varphi(x)\right|^{2}}\left(-r_{a}^{\prime}(\varphi(x))\right) \leqslant \frac{f_{1}(x)}{\left|\nabla_{a} \varphi(x)\right|^{2}} \quad \forall x \in \Omega \backslash\left\{x_{0}\right\} .
$$

This can be written:

$$
-r_{a}^{\prime \prime}(\varphi)\left|\nabla_{a} \varphi\right|^{2}-r_{a}^{\prime}(\varphi) \cdot A \varphi \leqslant f_{1} \quad \text { in } \Omega \backslash\left\{x_{0}\right\} .
$$

And since $r_{a}(\cdot)$ and $\varphi(\cdot)$ are regular, (18) is valid in all $\Omega$. Thus (18) means

$$
\left\{\begin{array}{l}
-A\left(r_{a}(\varphi)\right) \leqslant f_{1} \quad \text { in } \Omega, \\
\left.r_{a}(\varphi)\right|_{\partial \Omega}=0, \quad r_{a}(\varphi) \in W_{0}^{1, \infty}(\Omega)
\end{array}\right.
$$

which implies that $r_{A}(\cdot)=r_{a} \circ \varphi(\cdot)$ is a subsolution of Eq. $\left(E_{A}\right)$.

Second step. Regularization in the nonregular case.

If $Q_{1}^{a, \varphi}(t)$ and $k_{1}^{a, \varphi} / t$ are not regular, we use a regularization process. Let $Q_{\epsilon}(\cdot)$ a regular function such that $Q_{\epsilon}$ tends to $Q_{1}^{a, \varphi}$ in $L^{2}(0, T)$ strongly as $\epsilon$ goes to zero. From Appendix A there exists a regular function $k_{\epsilon}$ such that:

$$
\lim _{\epsilon} \int_{0}^{T}\left|k_{\epsilon}-k_{1}^{a, \varphi}\right|^{2} \mathrm{~d} t=0, \quad \lim _{\epsilon} \int_{0}^{T}\left|\frac{k_{\epsilon}-k}{t}\right| \mathrm{d} t=0 .
$$

Let us consider $r_{\epsilon}$ the solution of

$$
\left\{\begin{array}{l}
-r_{\epsilon}^{\prime \prime}(t)-\frac{Q_{\epsilon}(t)}{t+\epsilon} r_{\epsilon}^{\prime}(t)=\frac{k_{\epsilon}(t)}{t+\epsilon} \\
r_{\epsilon}^{\prime}(0)=0, \quad r_{\epsilon}(T)=0
\end{array}\right.
$$


$r_{\epsilon}(\cdot)$ is regular enough in order to define $r_{\epsilon}^{\prime}(\varphi(x))$ and $r_{\epsilon}^{\prime \prime}(\varphi(x))$ and it is easy to see that

$$
\left\{\begin{array}{l}
0 \leqslant r_{\epsilon}(t) \leqslant(T-t) \int_{0}^{T} \frac{k_{\epsilon}(s)}{s} \mathrm{~d} s \leqslant c, \\
0 \leqslant-r_{\epsilon}^{\prime}(t) \leqslant \int_{0}^{t} \frac{k_{\epsilon}(s)}{s} \mathrm{~d} s \leqslant \int_{0}^{T} \frac{k_{\epsilon}(s)}{s} \leqslant c .
\end{array}\right.
$$

Thus there is a subsequence, again labeled $\epsilon$, such that $r_{\epsilon}$ tends to $r_{a}$ in $W_{0}^{1, \infty}$ (]0,T [) for the weak-* topology, as $\epsilon$ goes to zero. Let us recall that $r_{a}(\cdot)$ is the solution of (13). From Appendix B we get

$$
-A\left(r_{a}(\varphi)\right) \leqslant \frac{k_{1}^{a, \varphi}(\varphi) \cdot\left|\nabla_{a} \varphi\right|^{2}}{\varphi} \text { in } H^{-1}(\Omega)
$$

which implies

$$
-A\left(r_{a}(\varphi)\right) \leqslant f_{1} \quad \text { in } H^{-1}(\Omega),
$$

with

$$
r_{a} \circ \varphi(\cdot)=r_{a}(\cdot) \in H_{0}^{1}(\Omega)
$$

using definition (6) of $k_{1}^{a, \varphi}$.

\subsection{Supersolution of equation}

$$
\left\{\begin{array}{l}
-B u_{B}=f_{2} \\
\left.u_{B}\right|_{\partial \Omega}=0 .
\end{array} \text { in } \Omega, b=\left(b_{i j}\right),\right.
$$

We proceed in the same way as in the previous section. Using operator $B$, we consider the similar equation of (13)

$$
\left\{\begin{array}{l}
\left.-r_{b}^{\prime \prime}(t)-\frac{Q_{N}^{b, \varphi}(t)}{t} r_{b}^{\prime}(t)=\frac{k_{2}^{b, \varphi}}{t} \quad \text { in }\right] 0, T[, \\
r_{b}^{\prime}(0)=0, \quad r_{b}(T)=0,
\end{array}\right.
$$

where $Q_{N}^{b, \varphi}(\cdot)$ and $k_{2}^{b, \varphi}(\cdot)$ are defined, respectively, in (5) and (7) Section 2 and $r_{b}(\cdot)$ is given by

$$
r_{b}(t)=\int_{t}^{T} \frac{1}{\beta_{b}(s)} \cdot \int_{0}^{s} \beta_{b}(\theta) \frac{k_{2}^{b, \varphi}(\theta)}{\theta} \mathrm{d} \theta \mathrm{d} s
$$

with $\beta_{b}(\cdot)$ satisfying

$$
\left\{\begin{array}{l}
\left.\frac{\beta_{b}^{\prime}(t)}{\beta_{b}(t)}=\frac{Q_{N}^{b, \varphi}(t)}{t} \text { in }\right] 0, T[ \\
\beta_{b}(0)=0
\end{array}\right.
$$

Proposition 8. Let us $\operatorname{set}_{B}(x)=r_{b} \circ \varphi(x)$. Then $r_{B}(\cdot)$ is a supersolution of (20).

The proof is similar to that of Proposition 7. 


\section{The comparison principle}

From operators $A$ and $B$ let us introduce the following kernels:

$$
\begin{aligned}
& K_{2}^{b, \varphi}(s, \theta)=\exp \left[-\int_{\theta}^{s} \frac{Q_{N}^{b, \varphi}(\sigma)}{\sigma} \mathrm{d} \sigma\right], \\
& K_{1}^{a, \varphi}(s, \theta)=\exp \left[-\int_{\theta}^{s} \frac{Q_{1}^{a, \varphi}(\sigma)}{\sigma} \mathrm{d} \sigma\right],
\end{aligned}
$$

where $Q_{1}^{a, \varphi}$ and $Q_{N}^{b, \varphi}$ are defined in (4) and (5) respectively.

To locally compare $u_{A}(\cdot)$ and $u_{B}(\cdot)$, it is necessary that there exists some links between $\left(A, f_{1}\right)$ and $\left(B, f_{2}\right)$. In this paper we give a sufficient condition to establish the following comparison principle:

$$
u_{A}(x)>u_{B}(x) \text { in } \Omega \text {. }
$$

Theorem 9. Let us assume that there exists a constant $c_{0}>0$ such that

$$
c_{0} \cdot \int_{t}^{T}\left[\int_{0}^{s} K_{1}^{a, \varphi}(s, \sigma) \frac{k_{1}^{a, \varphi}(\sigma)}{\sigma} \mathrm{d} \sigma\right] \mathrm{d} s \geqslant \int_{t}^{T}\left[\int_{0}^{s} K_{2}^{b, \varphi}(s, \sigma) \frac{k_{2}^{b, \varphi}(\sigma)}{\sigma} \mathrm{d} \sigma\right] \mathrm{d} s .
$$

Then under assumption (H1) the following comparison holds:

$$
c_{0} \cdot u_{A}(x)>u_{B}(x) \text { a.e. } x \in \Omega
$$

where $u_{A}(\cdot)$ and $u_{B}(\cdot)$ are, respectively, solution of (12) and (20).

Proof. Now this proof is easy to get. Indeed our assumption means that

$$
\left.c_{0} \cdot r_{a}(t) \geqslant r_{b}(t) \quad \forall t \in\right] 0, T[,
$$

and since $r_{a} \circ \varphi$ and $r_{b} \circ \varphi$ are respectively subsolution of $\left(E_{A}\right)$ and supersolution of $\left(E_{B}\right)$, we clearly obtain:

$$
c_{0} \cdot u_{A}(x)>c_{0} \cdot r_{a} \circ \varphi(x) \geqslant r_{b} \circ \varphi(x)>u_{B}(x) \text { in } \Omega \text {. }
$$

\section{Remarks.}

1. To prove the previous result, we assumed hypothesis (H1) i.e. we supposed that $k_{1}^{a, \varphi}(t) / t$ and $k_{2}^{b, \varphi}(t) / t$ belong to $L^{1}(0, T)$. This means, roughly speaking, that $f_{i}(x) / \varphi(x), i=1,2$, is bounded in a neighbourhood of $x_{0}$, where $x_{0}$ is the critical point of $\varphi$, which also satisfies $\varphi\left(x_{0}\right)=0$. If $k_{1}^{a, \varphi}(t) / t$ and $k_{2}^{b, \varphi}(t) / t$ do not belong to $L^{1}(0, T)$, we can proceed by approximation process. Let us mention briefly how to do. For any $\epsilon>0$, let us give a regular function $\psi_{\epsilon}$ such that:

$$
\begin{aligned}
& 0 \leqslant \psi_{\epsilon}(x) \leqslant 1 \quad \forall x \in \Omega, \\
& \psi_{\epsilon}(x)=1 \quad \forall x \in \Omega \backslash B\left(x_{0}, 2 \epsilon\right), \\
& \psi_{\epsilon}(x)=0 \quad \forall x \in \overline{B\left(x_{0}, \epsilon\right)},
\end{aligned}
$$

where $B\left(x_{0}, r\right)$ is the open ball centered at $x_{0}$, with radius $r>0$. Let us consider $u_{A}^{\epsilon}(\cdot)$ and $u_{B}^{\epsilon}(\cdot)$, solution respectively of

$$
\begin{array}{ll}
-A u_{A}^{\epsilon}=f_{1} \cdot \psi_{\epsilon} & \text { in } \Omega, u_{A}^{\epsilon} \in H_{0}^{1}(\Omega), \\
-B u_{B}^{\epsilon}=f_{2} \cdot \psi_{\epsilon} & \text { in } \Omega, u_{B}^{\epsilon} \in H_{0}^{1}(\Omega) .
\end{array}
$$


Now it is sufficient to compare $u_{A}^{\epsilon}$ and $u_{B}^{\epsilon}$ uniformly with respect to $\epsilon$ using Theorem 9 . The main difficulty is then to prove some estimates uniformly with respect to $\epsilon$. This point of our work is left to the interested reader: in this paper, our goal is to develop the main idea without peppering it with too many technical details.

2. We will see that in some important and particular cases the kernels $K_{1}^{a, \varphi}$ and $K_{2}^{b, \varphi}$ are more explicit than those given previously: cf. $\left(\mathrm{H}_{\mathrm{pr}}\right)$ and $\left(\mathrm{H}_{2}\right)$ Section 7 .

\section{The case $\Omega$ is an open regular, bounded and connected subset of $\mathbb{R}^{N}$}

In this section we assume that $\Omega$ is such that there exists an open bounded and regular convex, noted $\widetilde{\Omega}$, and a deformation $h=\left(h_{1}, \ldots, h_{N}\right)$ mapping $\Omega$ to $\widetilde{\Omega}$, satisfying $\operatorname{det} \nabla h(x)>0 \forall x \in \bar{\Omega}$. In order to use the previous results, our idea is to transport equation $\left(E_{A}\right)$ and $\left(E_{B}\right)$ to $\widetilde{\Omega}$ by applying $h$. Let us notice $\tilde{A}$ and $\widetilde{B}$ the transported operators which are variable coefficients; we have the following

Proposition 10. Let $A=-\sum_{i j} a_{i j} \frac{\partial^{2}}{\partial x_{i} \partial x_{j}}$ be the operator acting on $\Omega$, and $\tilde{A}$ its corresponding transported operator acting on $\widetilde{\Omega}$. Then we have:

$$
\left\{\begin{array}{l}
\tilde{A}=-\sum_{k \ell} \tilde{a}_{k \ell} \frac{\partial^{2}}{\partial y_{k} \partial y_{\ell}}+\sum_{k} c_{k} \frac{\partial}{\partial y_{k}}, \\
\tilde{a}_{k \ell}=\tilde{a}_{k \ell}(y)=\operatorname{Tr}\left(a \cdot\left(\nabla h_{\ell} \otimes \nabla h_{k}\right) \circ h^{-1}(y)\right), \\
c_{k}=c_{k}(y)=\operatorname{Tr}\left(a \cdot\left(D^{2} h_{k}\right) \circ h^{-1}(y)\right)
\end{array}\right.
$$

where $D^{2} h_{k}$ stands for the Hessien operator of $h_{k}$ and $\alpha \otimes \beta$ is the matrix $\left(\alpha_{i} \beta_{j}\right)$ for any $\alpha$ and $\beta$ belonging to $\mathbb{R}^{N}$.

The proof of this result is based on a simple computation and is left to the reader. The same result holds for $B$ and $\widetilde{B}$.

Remark. To study explicit examples, it is judicious to choose as much as possible the ball $B(0, R)$ as $\widetilde{\Omega}$. In fact the coefficients of operators $\tilde{A}$ and $\widetilde{B}$ are non constant and we have to minimize technical details by choosing the radial shape function.

It is easy to show that

Proposition 11. The operator $-\sum_{k \ell} \tilde{a}_{k \ell} \frac{\partial^{2}}{\partial y_{k} \partial y_{\ell}}$ is uniformly elliptic and such that $\tilde{a}_{k \ell}=\tilde{a}_{\ell k}$ for any $\ell, k$.

For any $j, 1 \leqslant j \leqslant N$, let us consider $\omega_{j}$ the $j$ th eigenfunction of matrix $\tilde{a}=\left(\tilde{a}_{i j}\right)$ corresponding to eigenvalue $\lambda_{j}(\tilde{a})$. Let us assume that $\left\|\omega_{j}\right\|=1$.

Proposition 12. Spectral estimates of matrix $\left(\tilde{a}_{i j}\right)$.

We have the following estimates:

$$
\lambda_{1}(a) \gamma_{1}^{2}(h) \leqslant \underline{\Lambda}_{j} \leqslant \lambda_{j}(\tilde{a}) \leqslant \bar{\Lambda}_{j} \leqslant \lambda_{N}(a) \gamma_{N}^{2}(h)
$$

where

$$
\begin{aligned}
& \underline{\Lambda}_{j}=\inf _{x \in \bar{\Omega}} \inf _{\sigma \in G_{N}} \sum_{i=1}^{N} \lambda_{\sigma(i)}(a)\left[\left({ }^{t} \nabla h(x) \omega_{j}\right)_{i}\right]^{2}=\operatorname{infinf}\left\|\sqrt{D_{a, \sigma}} t \nabla h \omega_{j}\right\|^{2}, \\
& \bar{\Lambda}_{j}=\sup _{x \in \bar{\Omega}} \sup _{\sigma \in G_{N}} \sum_{i=1}^{N} \lambda_{\sigma(i)}(a)\left[\left({ }^{t} \nabla h(x) \omega_{j}\right)_{i}\right]^{2}=\sup \sup \left\|\sqrt{D_{a, \sigma} t} \nabla h \omega_{j}\right\|^{2}
\end{aligned}
$$


$\gamma_{i}^{2}(h)=\lambda_{i}\left({ }^{t} \nabla h \cdot \nabla h\right)$ for $i=1, \ldots, N ; G_{N}$ is the permutation group of the set $\{1,2, \ldots, N\}$. The matrix ${ }^{t} \nabla h$ stands for the adjoint of the matrix $\nabla h$. In addition

$$
\lambda_{j}(\tilde{a})=\sum_{i} \lambda_{i}(a)\left[\left({ }^{t} \nabla h(x) \omega_{j}\right)_{i}\right]^{2}=\left\|\sqrt{D_{a}} \cdot{ }^{t} \nabla h(x) \omega_{j}\right\|^{2}
$$

with

$$
D_{a}=\left(\begin{array}{ccc}
\lambda_{1}(a) & \ldots & 0 \\
\vdots & \ddots & \vdots \\
0 & \ldots & \lambda_{N}(a)
\end{array}\right), \quad D_{a, \sigma}=\left(\begin{array}{ccc}
\lambda_{\sigma(1)}(a) & \ldots & 0 \\
\vdots & \ddots & \vdots \\
0 & \ldots & \lambda_{\sigma(N)}(a)
\end{array}\right)
$$

Proof. We have

$$
\lambda_{j}(\tilde{a})=\left(\tilde{a} \omega_{j}, \omega_{j}\right)=\sum_{k, \ell} \tilde{a}_{k, \ell}\left(\omega_{j}\right)_{k} \cdot\left(\omega_{j}\right)_{\ell} .
$$

Let us consider the following bilinear form

$$
a(\xi, \eta)=\sum_{i j} a_{i j} \xi_{j} \eta_{i}=\xi^{T} \cdot a \cdot \eta
$$

we have $\tilde{a}_{k, \ell}=a\left(\nabla h_{k}, \nabla h_{\ell}\right)$. Let us consider the basis $\left(e_{i}\right), i=1, \ldots, N$ of unitary eigenvectors of matrix $a=$ $\left(a_{i j}\right)$. We have

$$
\nabla h_{\ell}=\sum_{i} \alpha_{i}^{\ell} e_{i}, \quad \nabla h_{k}=\sum_{j} \alpha_{j}^{k} e_{j}
$$

Thus

$$
\tilde{a}_{k \ell}=\sum_{i j} \alpha_{j}^{k} \alpha_{i}^{\ell} a\left(e_{i}, e_{j}\right)=\sum_{i j} \alpha_{i}^{\ell} \alpha_{j}^{k} \lambda_{i}(a) \delta_{i j}=\sum_{i} \alpha_{i}^{\ell} \alpha_{i}^{k} \lambda_{i}(a)
$$

For any $\xi$ belonging to $\mathbb{R}^{N}$ we have:

$$
\sum_{k \ell} \tilde{a}_{k \ell} \cdot \xi_{k} \cdot \xi_{\ell}=\sum_{k \ell}\left(\sum_{i} \lambda_{i}(a) \alpha_{i}^{\ell} \alpha_{i}^{k}\right) \xi_{k} \xi_{\ell}=\sum_{i} \lambda_{i}(a)\left(\sum_{k} \alpha_{i}^{k} \xi_{k}\right)^{2}=\sum_{i} \lambda_{i}(a)\left[\left({ }^{t} \nabla h \xi\right)_{i}\right]^{2} .
$$

Now let us take $\xi=\omega_{j}$ in (2). Using (1), we obtain

$$
\lambda_{j}(\tilde{a})=\left(\tilde{a} \omega_{j}, \omega_{j}\right)=\sum_{i} \lambda_{i}(a)\left[\left({ }^{t} \nabla h \omega_{j}\right)_{i}\right]^{2}=\left\|\sqrt{D_{a}} \cdot{ }^{t} \nabla h \omega_{j}\right\|^{2} .
$$

The next parts of the proof is then obvious.

Propositions 12 and 3 give without too technical computations, a satisfactory spectral estimate for $\tilde{A}$. In fact we obtain the following

Corollary 13 (of Propositions 12 and 3). If we denote by $\varphi(\cdot)$ the shape function on $\widetilde{\Omega}$, we have

$$
\left\{\begin{array}{l}
\frac{Q_{N}^{\tilde{a}, \varphi}(t)}{t} \leqslant \frac{\tilde{A} \varphi}{\left|\nabla_{\tilde{a}} \varphi(x)\right|^{2}} \leqslant \frac{Q_{1}^{\tilde{a}, \varphi}(t)}{t} \\
\forall x \in\{y \in \Omega \mid \varphi(y)=t\}, \quad \forall t \in] 0, T]
\end{array}\right.
$$

where in $Q_{N}^{\tilde{c}, \varphi}$ defined in (3) and (5), $\lambda_{i}(\tilde{c})$ is replaced by $\underline{\Lambda_{i}}$, and in $Q_{1}^{\tilde{c}, \varphi}$ defined in (2) and (4), $\lambda_{i}(\tilde{c})$ is replaced by $\bar{\Lambda}_{i}$. 
Remark. If $\widetilde{\Omega}=B(0, R)$ and $\varphi(y)=|y|^{2}$ the previous result becomes:

$$
\frac{\sum_{i} \underline{\Lambda}_{i}}{\sup _{i} \bar{\Lambda}_{i}} \cdot \frac{1}{4 t} \leqslant \frac{\tilde{A} \varphi(y)}{\left|\nabla_{\tilde{a}} \varphi(y)\right|^{2}} \leqslant \frac{\sum_{i} \bar{\Lambda}_{i}}{\inf _{i} \underline{\Lambda}_{i}} \cdot \frac{1}{4 t}
$$

$\forall y$ such that $|y|^{2}=t$. Compare with Corollary 5 .

Remark (About the setting of the ordinary differential equation linked with $\tilde{A}$ ). The additional term $\sum_{k} c_{k} \frac{\partial}{\partial y_{k}}$, which appears in (0) Proposition 10, does not imply any major disadvantage for our method. In the ordinary differential equation (E) in Section 4, the coefficient $q(\cdot)$ of $\omega^{\prime}(\cdot)$ is the only one which changes: the term originating from $\sum_{k} c_{j} \frac{\partial}{\partial y_{k}}$ adds itself to the coefficient $q(\cdot)$. In order to avoid doing the same work again, we omit the work corresponding to Sections 4 and 5 . Theorem 9 is still valid in this framework.

\section{Examples and application}

Example 1. The significance of shape function $\varphi$ is illustrated by the following pseudo-radial example:

$$
\Omega=B(0, R) \quad \text { in } \mathbb{R}^{N} ; \quad a=\left(a_{i j}\right), \quad b=\left(b_{i j}\right)
$$

are constant matrices; $f_{i}(x)=f_{i}\left(|x|^{2}\right), i=1,2 ; \varphi(x)=|x|^{2} ; T=R^{2} ; c_{0}=1$,

$$
\frac{\operatorname{Tr}(c)}{2 \lambda_{N}(c)} \cdot \frac{1}{\varphi(x)} \leqslant \frac{(C \varphi)(x)}{\left|\nabla_{c} \varphi(x)\right|^{2}} \leqslant \frac{\operatorname{Tr}(c)}{2 \lambda_{1}(c)} \cdot \frac{1}{\varphi(x)},
$$

where $C$ stands for $A$ or $B$.

$$
\frac{f_{i}(\varphi(x))}{4 \varphi(x)} \cdot \frac{1}{\lambda_{N}(c)} \leqslant \frac{f_{i}\left(|x|^{2}\right)}{\left|\nabla_{c} \varphi(x)\right|^{2}} \leqslant \frac{f_{i}(\varphi(x))}{4 \varphi(x)} \cdot \frac{1}{\lambda_{1}(c)}, \quad i=1,2 .
$$

In this case our assumption $(\mathrm{H})$ becomes:

$$
\begin{aligned}
r_{a}(y) & =\int_{y}^{R^{2}} \frac{1}{4 \lambda_{N}(a)}\left[\int_{0}^{t}\left(\frac{s}{t}\right)^{\operatorname{Tr}(a) /\left(2 \lambda_{1}(a)\right)} \frac{f_{1}(s)}{s} \mathrm{~d} s\right] \mathrm{d} t \\
& \geqslant \int_{y}^{R^{2}} \frac{1}{4 \lambda_{1}(b)}\left[\int_{0}^{t}\left(\frac{s}{t}\right)^{\operatorname{Tr}(b) /\left(2 \lambda_{N}(b)\right)} \frac{f_{2}(s)}{s} \mathrm{~d} s\right] \mathrm{d} t=r_{b}(y),
\end{aligned}
$$

and we obtain:

$$
u_{A}(x)>u_{B}(x) \text { a.e. } x \in \Omega \text {. }
$$

\section{Remarks.}

1. Let us point out that to well define the above hypothesis it is sufficient to assume, for instance, that $f_{i}, i=1,2$, is bounded on some neighbourhood of $O$.

2. In the particular radial case i.e. where $a=b=\left(\delta_{i j}\right),\left(\mathrm{H}_{\mathrm{pr}}\right)$ becomes:

$$
r_{a}(y)=\frac{1}{4} \int_{y}^{R^{2}} \mathrm{~d} t \int_{0}^{t}\left(\frac{s}{t}\right)^{N / 2} \frac{f_{1}(s)}{s} \mathrm{~d} s \geqslant \frac{1}{4} \int_{y}^{R^{2}} \mathrm{~d} t \int_{0}^{t}\left(\frac{s}{t}\right)^{N / 2} \frac{f_{2}(s)}{s} \mathrm{~d} s=r_{b}(y) .
$$


After a change of variables it is easy to show that

$$
u_{1}(x)=r_{a}\left(|x|^{2}\right), \quad u_{2}(x)=r_{b}\left(|x|^{2}\right)
$$

are respectively the solution of

$$
\left\{\begin{array}{l}
-\Delta u_{i}=f_{i} \quad \text { in } B(0, R) \\
\left.u_{i}\right|_{\partial B}=0, \quad i=1,2 .
\end{array}\right.
$$

And then $\left(\mathrm{H}_{\mathrm{pr}}\right)$ is the optimal condition to compare $u_{1}(x)$ and $u_{2}(x)$ [9]. This very particular example shows, if necessary, that our spectral estimates (SE) and consequently our hypothesis $(\mathrm{H})$ are in some sense optimal: the possible loss of the optimal quality of the result is a consequence of the choice of the shape function $\varphi$.

To understand inequality $\left(\mathrm{H}_{\mathrm{pr}}\right)$ we think that it is interesting to give a sufficient inequality less sharp than $\left(\mathrm{H}_{\mathrm{pr}}\right)$ but more explicit than $\left(\mathrm{H}_{\mathrm{pr}}\right)$.

Proposition 14. Assume that $\left.\alpha\left(f_{1}\right)=\inf \left\{f_{1}\left(|x|^{2}\right) \mid x \in \overline{B(0, R)}\right\}>0\right\}$ and

$$
\frac{\lambda_{1}(a)}{\lambda_{N}(a)} \cdot \frac{\alpha\left(f_{1}\right)}{\operatorname{Tr}(a)+2 \lambda_{1}(a)} \geqslant \frac{\lambda_{N}(b)}{\lambda_{1}(b)} \cdot \frac{\beta\left(f_{2}\right)}{\operatorname{Tr}(b)+2 \lambda_{N}(b)}
$$

where $\beta\left(f_{2}\right)=\sup \left\{f_{2}\left(|x|^{2}\right) \mid x \in B \overline{(0, R)}\right\}$. Then $\left(\mathrm{H}_{\mathrm{pr}}\right)$ holds.

Proof. It suffices to see that we have

$$
r_{a}(y) \geqslant \frac{\lambda_{1}(a) \alpha\left(f_{1}\right) \cdot\left(R^{4}-y^{2}\right)}{\lambda_{N}(a)\left(\operatorname{Tr}(a)+2 \lambda_{1}(a)\right)}, \quad \forall y \in\left[0, R^{2}\right],
$$

and

$$
r_{b}(y) \leqslant \frac{\lambda_{N}(b) \beta\left(f_{2}\right) \cdot\left(R^{4}-y^{2}\right)}{\lambda_{1}(b)\left(\operatorname{Tr}(b)+2 \lambda_{N}(b)\right)}, \quad \forall y \in\left[0, R^{2}\right] .
$$

Remark. Let $f_{1}\left(|x|^{2}\right)$ and $f_{2}\left(|x|^{2}\right)$ be two functions defined on $B(0, R)$. Assume $\alpha\left(f_{1}\right)>0$. Let $a_{0}$ and $b_{0}$ be two Lipschitzian positive matrices. Then there exists some constant $t_{0}>0$ such that for any $t \geqslant t_{0}$ inequality $(*)$ holds for $a=a_{0}$ and $b=t \cdot b_{0}$.

Example 2 (Ellipsoïdal case). Let us consider

$$
\Omega=\left\{x \in \mathbb{R}^{N}: \varphi(x)<T\right\}
$$

where $\varphi(x)=\sum_{i=1}^{N} m_{i} x_{i}^{2}, m_{i}>0 \forall i=1, \ldots, N$. We suppose $f_{i}(x)=f_{i}(\varphi(x))$ and $a=\left(a_{i j}\right), b=\left(b_{i j}\right)$ are constant matrices.

Proposition 15. Let $c=\left(c_{i j}\right)$ a symmetric positive matrix. Then the matrix $d=\left(d_{i j}\right)$, where $d_{i j}=c_{i j} m_{i} m_{j}$, is a symmetric positive matrix. This matrix is denoted $c \otimes(m \otimes m)$ where $m=\left(m_{1}, m_{2}, \ldots, m_{N}\right)$ belongs to $\mathbb{R}^{N}$.

Proof. Let us remark that, for any $x \in \mathbb{R}^{N}, x \neq 0$, we have:

$$
\frac{(d x, x)}{|x|^{2}}=\frac{\sum_{i j} c_{i j}\left(x_{i} m_{i}\right) \cdot\left(x_{j} m_{j}\right)}{\sum_{i}\left(x_{i} m_{i}\right)^{2}} \cdot \frac{\sum_{i}\left(m_{i}^{2} x_{i}^{2}\right)}{|x|^{2}} .
$$

Then it is clear that

$$
\lambda_{1}(d) \geqslant \lambda_{1}(c) \cdot\left[\inf m_{i}^{2} \mid i=1, \ldots, N\right]>0 .
$$

The result follows from that fact that $\lambda_{1}(d)$ is the lowest eigenvalue of $d$. 
The spectral estimate (SE) becomes from Corollary 4

$$
\frac{\Lambda_{N}(c, \varphi)}{2 \lambda_{N}(c \otimes(m \otimes m))} \cdot \underline{m} \cdot \frac{1}{\varphi(x)} \leqslant \frac{(C \varphi)(x)}{\left|\nabla_{c} \varphi(x)\right|^{2}} \leqslant \frac{\Lambda_{1}(c, \varphi)}{2 \lambda_{1}(c \otimes(m \otimes m))} \cdot \bar{m} \cdot \frac{1}{\varphi(x)}
$$

where $\underline{m}=\inf \left[m_{i} \mid i=1, \ldots, N\right], \bar{m}=\sup \left[m_{i} \mid i=1, \ldots, N\right], \Lambda_{N}(c, \varphi)$ and $\Lambda_{1}(c, \varphi)$ are defined as in Corollary 4:

$$
\Lambda_{1}(c, \varphi)=\sup _{\sigma \in G_{N}} \sum_{i} \lambda_{i}(c) m_{\sigma(i)}, \quad \Lambda_{N}(c, \varphi)=\inf _{\sigma \in G_{N}} \sum_{i} \lambda_{i}(c) m_{\sigma(i)}
$$

In addition we have:

$$
\frac{f_{i}(\varphi)}{4 \varphi} \cdot \frac{1}{\lambda_{N}(c)} \leqslant \frac{f_{i}(\varphi(x))}{\left|\nabla_{c} \varphi(x)\right|^{2}} \leqslant \frac{f_{i}(\varphi)}{4 \varphi} \cdot \frac{1}{\lambda_{1}(c)}, \quad i=1,2 .
$$

Thus condition (H1) becomes:

$$
\begin{aligned}
& \int_{y}^{T} \frac{1}{\lambda_{N}(a)}\left[\int_{0}^{t}\left(\frac{s}{t}\right)^{\Lambda_{1}(a, \varphi) \cdot \bar{m} /\left(2 \lambda_{1}(a \otimes m \otimes m)\right)} \cdot \frac{f_{1}(s)}{s} \mathrm{~d} s\right] \mathrm{d} t \\
& \geqslant \int_{y}^{T} \frac{1}{\lambda_{1}(b)}\left[\int_{0}^{t}\left(\frac{s}{t}\right)^{\Lambda_{N}(b, \varphi) \cdot \underline{m} /\left(2 \lambda_{N}(b \otimes m \otimes m)\right)} \cdot \frac{f_{2}(s)}{s} \mathrm{~d} s\right] \mathrm{d} t .
\end{aligned}
$$

\subsection{Applications}

\subsubsection{A priori estimates in linear case}

Let us consider a sequence of problems

$$
\left\{\begin{array}{l}
-\sum_{i j} a_{i j}^{\epsilon}(x) \frac{\partial^{2} u_{\epsilon}}{\partial x_{i} \partial x_{j}}=f\left(|x|^{2}\right) \geqslant 0 \quad \text { in } \Omega=B(0, R) \\
\left.u_{\epsilon}\right|_{\partial B}=0
\end{array}\right.
$$

where the coefficients $a_{i j}^{\epsilon}(\cdot)$ are regular. Let us assume that there exists two constant, symmetric, definite positive matrices $c$ and $d$ such that

$$
\begin{aligned}
& \left\{\begin{array}{l}
\frac{\operatorname{Tr}\left(a^{\epsilon}(x)\right)}{2 \lambda_{N}\left(a^{\epsilon}(x)\right)} \geqslant \frac{\operatorname{Tr}(c)}{2 \lambda_{N}(c)} \quad \forall x \in B(0, R), \\
\lambda_{1}\left(a^{\epsilon}(x)\right) \geqslant \lambda_{1}(c) \quad \forall x \in B(0, R) ;
\end{array}\right. \\
& \left\{\begin{array}{l}
\frac{\operatorname{Tr}\left(a^{\epsilon}(x)\right)}{2 \lambda_{1}\left(a^{\epsilon}(x)\right)} \leqslant \frac{\operatorname{Tr}(d)}{2 \lambda_{1}(d)} \\
\lambda_{N}\left(a^{\epsilon}(x)\right) \leqslant \lambda_{N}(d)
\end{array} \quad \forall x \in B(0, R),\right.
\end{aligned}
$$

The result is:

(i) if (H1) holds then we have

$$
u_{\epsilon}(x) \leqslant s\left(|x|^{2}\right) \quad \forall x \in B(0, R),
$$

(ii) if (H2) holds then we have

$$
u_{\epsilon}(x) \geqslant r\left(|x|^{2}\right) \quad \forall x \in B(0, R)
$$

where 


$$
\begin{aligned}
& s\left(|x|^{2}\right)=\int_{|x|^{2}}^{R^{2}} \frac{1}{4 \lambda_{1}(c)}\left[\int_{0}^{t}\left(\frac{s}{t}\right)^{\operatorname{Tr}(c) /\left(2 \lambda_{N}(c)\right)} \frac{f(s)}{s} \mathrm{~d} s\right] \mathrm{d} t, \\
& r\left(|x|^{2}\right)=\int_{|x|^{2}}^{R^{2}} \frac{1}{4 \lambda_{N}(d)}\left[\int_{0}^{t}\left(\frac{s}{t}\right)^{\operatorname{Tr}(d) /\left(2 \lambda_{1}(d)\right)} \frac{f(s)}{s} \mathrm{~d} s\right] \mathrm{d} t .
\end{aligned}
$$

\subsubsection{Comparison in nonlinear case}

Let us consider the two nonlinear equations

$$
\begin{aligned}
& \left\{\begin{array}{l}
-\sum_{i j} a_{i j}(u) \frac{\partial^{2} u}{\partial x_{i} \partial x_{j}}=f(|x|)^{2} \quad \text { in } \Omega=B(0, R), \\
\left.u\right|_{\partial B}=0,
\end{array}\right. \\
& \left\{\begin{array}{l}
-\sum_{i j} b_{i j}(v) \frac{\partial^{2} v}{\partial x_{i} \partial x_{j}}=g(|x|)^{2} \quad \text { in } \Omega=B(0, R), \\
\left.u\right|_{\partial B}=0 .
\end{array}\right.
\end{aligned}
$$

Assume that there exists two constant, symmetric, definite positive matrices $c$ and $d$ such that:

$$
\begin{aligned}
& \left\{\begin{array}{l}
\frac{\operatorname{Tr} a(t)}{2 \lambda_{N}(a(t))} \geqslant \frac{\operatorname{Tr} c}{2 \lambda_{N}(c)} \quad \forall t \in \mathbb{R}, \\
\lambda_{1}(a(t)) \geqslant \lambda_{1}(c) \quad \forall t \in \mathbb{R},
\end{array}\right. \\
& \left\{\begin{array}{l}
\frac{\operatorname{Tr} b(t)}{2 \lambda_{1}(b(t))} \leqslant \frac{\operatorname{Tr}(d)}{2 \lambda_{1}(d)} \\
\lambda_{N}(b(t)) \leqslant \lambda_{N}(d) .
\end{array} \quad \forall t \in \mathbb{R},\right.
\end{aligned}
$$

Then we have:

$$
u(x)<v(x) \quad \forall x \in B(0, R)
$$

if the following inequality is satisfied

$$
\begin{aligned}
& \int_{|x|^{2}}^{R^{2}} \frac{1}{4 \lambda_{1}(c)}\left[\int_{0}^{t}\left(\frac{s}{t}\right)^{\operatorname{Tr}(c) /\left(2 \lambda_{N}(c)\right)} \cdot \frac{f(s)}{s} \mathrm{~d} s\right] \mathrm{d} t \\
& \quad \leqslant \int_{|x|^{2}}^{R^{2}} \frac{1}{4 \lambda_{N}(d)}\left[\int_{0}^{t}\left(\frac{s}{t}\right)^{\operatorname{Tr}(d) /\left(2 \lambda_{1}(d)\right)} \cdot \frac{g(s)}{s} \mathrm{~d} s\right] \mathrm{d} t, \quad \forall x \in B(0, R) .
\end{aligned}
$$

\subsubsection{Elliptic problem setting in $\mathbb{R}^{N}$}

Asymptotic behaviour of the solution as $|x|$ goes to infinity. We suppose $N \geqslant 3$. We are going to study the asymptotic behaviour of the solution $u(\cdot)$ of the following equation:

$$
\left\{\begin{array}{l}
-\sum_{i j} a_{i j}(x) \frac{\partial^{2} u}{\partial x_{i} \partial x_{j}}=f(x) \quad \text { on } \mathbb{R}^{N}, \\
\lim _{|x| \rightarrow+\infty} u(x)=0,
\end{array}\right.
$$


where $f$ is a nonnegative function belonging to $L^{1}\left(\mathbb{R}^{N}\right)$ and having a compact support; we assume that $u$ exists. The symmetric matrix $a=\left(a_{i j}\right)$ is a bounded, Lipschitz and uniformly elliptic. We suppose that there exist two radial nonnegative functions $g$ and $h$ with compact support and satisfying:

$$
0 \leqslant g\left(|x|^{2}\right) \leqslant f(x) \leqslant h\left(|x|^{2}\right) \quad \forall x \in \mathbb{R}^{N} .
$$

We assume that $h\left(|x|^{2}\right)$ belongs to $L^{1}\left(\mathbb{R}^{N}\right)$.

Our idea is to approach, in the ball $B(0, R)$, the solution $u(\cdot)$ by the solution $u_{R}(\cdot)$ of:

$$
\left\{\begin{array}{l}
-\sum_{i j} a_{i j}(x) \frac{\partial^{2} u_{R}}{\partial x_{i} \partial x_{j}}=f(x) \quad \text { on } B(0, R), \\
\left.u_{R}\right|_{\partial B}=0
\end{array}\right.
$$

as $R$ goes to infinity. The previous comparison results permit us to estimate $u_{R}(\cdot)$ from above and from below by two known radial functions $v_{R}(\cdot)$ and $\omega_{R}(\cdot)$ respectively. As the behaviour of $v_{R}(\cdot)$ and $\omega_{R}(\cdot)$ are known we can deduce the behaviour of $u(\cdot)$ as $R$ goes to infinity. We shall see that the behaviour of the solution $u(\cdot)$ of (3) is controlled by the behaviour of the function

$$
\Lambda(x)=\frac{\operatorname{Tr}(a(x))}{\left(2 /|x|^{2}\right) \sum_{i j} a_{i j}(x) x_{i} x_{j}}
$$

as $|x|$ goes to infinity. This result is new; and it seems surprising for us. Let us set:

$$
\left\{\begin{array}{l}
\bar{\theta}=\lim _{R \rightarrow+\infty} \sup _{|x| \geqslant R} \Lambda(x) \\
\underline{\theta}=\lim _{R \rightarrow+\infty} \inf _{|x| \geqslant R} \Lambda(x) .
\end{array}\right.
$$

Since the matrix $a(\cdot)$ is bounded and uniformly elliptic, we have $0<\underline{\theta} \leqslant \bar{\theta}, \bar{\theta} \in \mathbb{R}^{+}$. We assume the following hypothesis:

(H3) there exist some positive radial function $\gamma(\cdot)$ such that

$$
\lim _{R \rightarrow+\infty} \sup _{|x| \geqslant R}\left|\Lambda(x)-\gamma\left(|x|^{2}\right)\right|=0 .
$$

Remark. It is interesting to note that we have the following estimates: for any $x$

$$
\frac{N}{2} \frac{\lambda_{1}(a(x))}{\lambda_{N}(a(x))} \leqslant \frac{\sum_{i=1}^{N} \lambda_{i}(a(x))}{2 \lambda_{N}(a(x))} \leqslant \Lambda(x) \leqslant \frac{\sum_{i=1}^{N} \lambda_{i}(a(x))}{2 \lambda_{1}(a(x))} \leqslant \frac{N}{2} \frac{\lambda_{N}(a(x))}{\lambda_{1}(a(x))} .
$$

The interval

$$
I(a)=\left[\sum_{i} \lambda_{i}(a) \mid 2 \lambda_{N}(a), \sum_{i}\left(\lambda_{i}(a) \mid 2 \lambda_{1}(a)\right]\right.
$$

measures the dispersion spectrum of the matrix $\left(a_{i j}\right)$. And we will see that the function $\Lambda(\cdot)$ leaving in $I(a)$ controls, in some sense, the behaviour of the solution of (3) as $|x|$ goes to infinity.

There exist two constant elliptic matrices $c$ and $d$ such that for any $x \in \mathbb{R}^{N}, \operatorname{Tr}(c) /\left(2 \lambda_{N}(c)\right) \leqslant \Lambda(x) \leqslant$ $\operatorname{Tr}(d) /\left(2 \lambda_{1}(d)\right)$. For any $\epsilon>0$ there exist $R_{\epsilon}>0$ such that for any $x$ such that $|x| \geqslant R_{\epsilon}$ we have:

$$
\underline{\theta}-\epsilon \leqslant \Lambda(x) \leqslant \bar{\theta}+\epsilon .
$$


For any $\epsilon>0$, let us set

$$
\bar{q}_{\epsilon}(t)=\frac{1}{t} \begin{cases}\bar{\theta}+\epsilon, & \text { if } t \geqslant\left(R_{\epsilon}+1\right)^{2}, \\ \bar{\ell}(t), & \text { if } R_{\epsilon}^{2} \leqslant t \leqslant\left(R_{\epsilon}+1\right)^{2}, \\ \frac{\operatorname{Tr}(d)}{2 \lambda_{1}(d)}, & \text { if } 0 \leqslant t \leqslant R_{\epsilon}^{2},\end{cases}
$$

where $\bar{\ell}(\cdot)$ is an affine function such that $\bar{q}_{\epsilon}(\cdot)$ is continuous. From (7) we have

$$
\Lambda(x) \leqslant \bar{q}_{\epsilon}\left(|x|^{2}\right) \quad \forall x \in \mathbb{R}^{N} .
$$

Similarly we define

$$
\underline{q}_{\epsilon}(t)=\frac{1}{t} \begin{cases}\underline{\theta}-\epsilon, & \text { if } t \geqslant\left(R_{\epsilon}+1\right)^{2}, \\ \underline{\ell}(t), & \text { if } R_{\epsilon}^{2} \leqslant t \leqslant\left(R_{\epsilon}+1\right)^{2}, \\ \frac{\operatorname{Tr}(c)}{2 \lambda_{N}(c)}, & \text { if } 0 \leqslant t \leqslant R_{\epsilon}^{2},\end{cases}
$$

where $\underline{\ell}(\cdot)$ is an affine function such that $\underline{q}_{\epsilon}(\cdot)$ is continuous. From (9) we have

$$
\underline{q}_{\epsilon}\left(|x|^{2}\right) \leqslant \Lambda(x) \quad \forall x \in \mathbb{R}^{N} .
$$

Proposition 18. Suppose that $a$ is a bounded Lipschitz and uniformly elliptic matrix. Then we have:

$$
0 \leqslant v_{\rho}^{\epsilon}\left(|x|^{2}\right) \leqslant u_{\rho}(x) \leqslant \omega_{\rho}^{\epsilon}\left(|x|^{2}\right) \quad \forall x \in B(0, \rho),
$$

where $v_{\rho}^{\epsilon}(\cdot)$ and $\omega_{\rho}^{\epsilon}(\cdot)$ are the following radial functions

$$
\begin{aligned}
& v_{\rho}^{\epsilon}(t)=\int_{t}^{\rho^{2}}\left[\int_{0}^{s} \exp \left[-\int_{\theta}^{s} \underline{q}_{\epsilon}(\sigma) \mathrm{d} \sigma\right] \cdot \frac{g(\theta)}{\theta} \mathrm{d} \theta\right] \mathrm{d} s, \\
& \omega_{\rho}^{\epsilon}(t)=\int_{t}^{\rho^{2}}\left[\int_{0}^{s} \exp \left[-\int_{\theta}^{s} \bar{q}_{\epsilon}(\sigma) \mathrm{d} \sigma\right] \cdot \frac{h(\theta)}{\theta} \mathrm{d} \theta\right] \mathrm{d} s .
\end{aligned}
$$

Proof. Let us consider the solution $v_{\rho}^{\epsilon}(\cdot)$ of

$$
\left\{\begin{array}{l}
\left.-v_{\rho}^{\epsilon^{\prime \prime}}(t)-\bar{q}_{\epsilon}(t) v_{\rho}^{\epsilon^{\prime}}(t)=g(t) \quad \text { on }\right] 0 \rho^{2}[, \\
v_{\rho}^{\epsilon^{\prime}}(0)=0, \quad v_{\rho}^{\epsilon}\left(\rho^{2}\right)=0, \quad \rho>R_{\epsilon} .
\end{array}\right.
$$

It is easy to see that

$$
v_{\rho}^{\epsilon}(t)=\int_{t}^{\rho^{2}}\left[\int_{0}^{s} \exp \left[-\int_{\theta}^{s} \bar{q}_{\epsilon}(\sigma) \mathrm{d} \sigma\right] \frac{g(\theta)}{\theta} \mathrm{d} \theta\right] \mathrm{d} s .
$$

From (5), (7), (8) and Theorem 9 we obtain

$$
0 \leqslant v_{\rho}^{\epsilon}\left(|x|^{2}\right) \leqslant u_{\rho}(x) \quad \forall x \in B(0, \rho) .
$$

By a similar way we can state that

$$
u_{\rho}(x) \leqslant \omega_{\rho}^{\epsilon}\left(|x|^{2}\right) \quad \forall x \in B(0, \rho) .
$$


It is clear that there exist two constants $\delta_{1}>\delta_{2}>0$ such that

$$
1<\delta_{1} \leqslant t \cdot \underline{q}_{\epsilon}(t) \leqslant t \cdot \bar{q}_{\epsilon}(t) \leqslant \delta_{2} \quad \forall t .
$$

From (11) and Proposition 18 we deduce the following result.

\section{Corollary 19.}

$$
0 \leqslant u_{\rho}(x) \leqslant \omega_{\rho}^{\epsilon}\left(|x|^{2}\right) \leqslant m\left(|x|^{2}\right)=\int_{|x|^{2}}^{+\infty}\left[\int_{0}^{s}\left(\frac{\theta}{s}\right)^{\delta_{1}} \frac{h(\theta)}{\theta} \mathrm{d} \theta\right] \mathrm{d} s .
$$

Proposition 20. We have

$$
0 \leqslant v_{\epsilon}\left(|x|^{2}\right) \leqslant u(x) \leqslant \omega_{\epsilon}\left(|x|^{2}\right) \quad \forall x \in \mathbb{R}^{N},
$$

where

$$
\begin{aligned}
& v_{\epsilon}\left(|x|^{2}\right)=\int_{|x|^{2}}^{+\infty}\left[\int_{0}^{s} \exp \left[-\int_{0}^{s} \bar{q}_{\epsilon}(\sigma) \mathrm{d} \sigma\right] \frac{g(\theta)}{\theta} \mathrm{d} \theta\right] \mathrm{d} s, \\
& \omega_{\epsilon}\left(|x|^{2}\right)=\int_{|x|^{2}}^{+\infty}\left[\int_{0}^{s} \exp \left[-\int_{0}^{s} \underline{q}_{\epsilon}(\sigma) \mathrm{d} \sigma\right] \frac{h(\theta)}{\theta} \mathrm{d} \theta\right] \mathrm{d} s .
\end{aligned}
$$

Proof. (1) first step. A priori estimates of $u_{\rho}(\cdot)$

Since the matrix $a(\cdot)$ is Lipschitz, (5) can be written:

$$
\left\{\begin{array}{l}
-\operatorname{div}\left(a \cdot \nabla u_{\rho}\right)+b \cdot \nabla u_{\rho}=f \quad \text { on } B(0, \rho), \\
\left.u_{\rho}\right|_{\partial B}=0
\end{array}\right.
$$

where

$$
b=\left(\begin{array}{c}
b_{1} \\
\vdots \\
b_{N}
\end{array}\right)
$$

belongs to $\left(L^{\infty}\left(\mathbb{R}^{N}\right)\right)^{N}$, with

$$
b_{j}=-\sum_{i=1}^{N} \frac{\partial a_{i j}}{\partial x_{i}} \text {. }
$$

Multiplying (12) by $u_{\rho}$ and after an integration on $B(0, \rho)$, it follows from the fact that $\left.b \in L^{\infty}\left(\mathbb{R}^{N}\right)\right)^{N}$ :

$$
\left\{\begin{array}{l}
0 \leqslant u_{\rho}(x) \leqslant c \quad \forall x \in \mathbb{R}^{N}, \\
\left\|\nabla u_{\rho}\right\|_{L^{2}(B)} \leqslant c
\end{array}\right.
$$

where $c$ stands for some constant which is independent of $\rho$ from Corollary 19 and where we have extended $u_{\rho}(\cdot)$ by zero outside $B(0, \rho)$. Up to a subsequence, labelled again $u_{\rho}(\cdot), u_{\rho}$ tends weakly to $u$ in $H_{\text {loc }}^{1}\left(\mathbb{R}^{N}\right)$, strongly in $L_{\text {loc }}^{2}\left(\mathbb{R}^{N}\right)$ and a.e. $x \in \mathbb{R}$ as $\rho$ goes to infinity. Since $f$ is nonnegative, from maximum principle ([4], [6]) the sequence $\rho \rightarrow u_{\rho}(\cdot)$ is increasing. Thus for any $x \in \mathbb{R}^{N}$, we have

$$
0 \leqslant \lim _{\rho \rightarrow+\infty} u_{\rho}(x)=u(x) \leqslant m\left(|x|^{2}\right),
$$


using Corollary 19. Thanks to (12) and (13) and up to a subsequence, $u_{\rho}(\cdot)$ converges to $u$ which is a solution of (3).

(2) second step. Limit of $v_{\rho}^{\epsilon}(\cdot)$ and $\omega_{\rho}^{\epsilon}(\cdot)$ as $\rho$ goes to infinity.

For any $\epsilon>0$ the sequences of functions $\rho \rightarrow v_{\rho}^{\epsilon}(\cdot)$ and $\rho \rightarrow \omega_{\rho}^{\epsilon}(\cdot)$ are increasing. Thus from Proposition 18 and Corollary 19 we have, for any $x \in \mathbb{R}^{N}$

$$
\lim _{\rho} v_{\rho}^{\epsilon}\left(|x|^{2}\right)=v_{\epsilon}\left(|x|^{2}\right), \quad \lim _{\rho} \omega_{\rho}^{\epsilon}\left(|x|^{2}\right)=\omega_{\epsilon}\left(|x|^{2}\right) .
$$

So $v_{\epsilon}\left(|x|^{2}\right) \leqslant u(x) \leqslant \omega_{\epsilon}\left(|x|^{2}\right) \forall x \in \mathbb{R}^{N}$.

Theorem 21. There exist $R>1$ such that:

$$
\forall x \in \mathbb{R}^{N}, \quad|x| \geqslant R, \quad 0 \leqslant c_{1} \hat{v}\left(|x|^{2}\right) \leqslant u(x) \leqslant c_{2} \widehat{\omega}\left(|x|^{2}\right)
$$

where $c_{i}, i=1,2$, are some positive constants and

$$
\begin{aligned}
& \hat{v}\left(|x|^{2}\right)=\frac{1}{|x|^{2(\bar{\theta}-1)},} \\
& \widehat{\omega}\left(|x|^{2}\right)=\frac{1}{|x|^{2(\underline{\theta}-1)}},
\end{aligned}
$$

where $\bar{\theta}$ and $\underline{\theta}$ are given by (5.1).

Proof. Our idea is to estimate from above $\omega_{\epsilon}(\cdot)$ and from below $v_{\epsilon}(\cdot)$.

(i) first step. Estimate from above of $\omega_{\epsilon}\left(|x|^{2}\right)$.

Let us consider (9) and $R_{\epsilon}$ defined in (6). Let us consider $R>R_{\epsilon}+1$, large enough such that $\operatorname{supp} h(\cdot) \subset\left[0, R^{2}[\right.$. The function $\omega_{\epsilon}\left(|x|^{2}\right)$ can be written for $|x|>R$

$$
\omega_{\epsilon}\left(|x|^{2}\right)=\int_{|x|^{2}}^{+\infty}\left[\int_{0}^{s} \chi_{[0, s]}(\theta) \exp \left(-\int_{\theta}^{R^{2}} \underline{q}_{\epsilon}(\sigma) \mathrm{d} \sigma\right) \exp \left(-\int_{R^{2}}^{s} \frac{\theta}{\sigma} \mathrm{d} \sigma\right) \frac{h(\theta)}{\theta} \mathrm{d} \theta\right] \mathrm{d} s,
$$

from the very definition of $\underline{q}_{\epsilon}(\cdot)$. Or again

$$
\omega_{\epsilon}\left(|x|^{2}\right)=\int_{|x|^{2}}^{+\infty}\left[\left(\frac{R^{2}}{s}\right)^{\underline{\theta}-\epsilon} \int_{0}^{s} \chi_{[0, s]}(\theta) \exp \left(-\int_{\theta}^{R^{2}} \underline{q}_{\epsilon}(\sigma) \mathrm{d} \sigma\right) \frac{h(\theta)}{\theta} \mathrm{d} \theta\right] \mathrm{d} s=\int_{|x|^{2}}^{+\infty}\left(\frac{R^{2}}{s}\right)^{\underline{\theta}-\epsilon} \cdot c(s) \mathrm{d} s .
$$

But since $\operatorname{supp} h(\cdot) \subset\left[0, R^{2}[\right.$, we have

$$
\begin{aligned}
c(s) & =\int_{0}^{s} \chi_{[0, s]}(\theta) \exp \left(-\int_{\theta}^{R^{2}} \underline{q} \epsilon(\sigma) \mathrm{d} \sigma\right) \frac{h(\theta)}{\theta} \mathrm{d} \theta \leqslant \int_{0}^{+\infty} \chi_{\left[0, R^{2}\right]}(\theta) \exp \left(-\int_{\theta}^{R^{2}} \underline{q}_{\epsilon}(\sigma) \mathrm{d} \sigma\right) \frac{h(\theta)}{\theta} \mathrm{d} \theta \\
& \leqslant \int_{0}^{+\infty} \chi_{\left[0, R^{2}\right]}(\theta) \exp \left(-\int_{\theta}^{R^{2}} \frac{\delta_{1}}{\sigma} \mathrm{d} \sigma\right) \frac{h(\theta)}{\theta} \mathrm{d} \theta
\end{aligned}
$$

because we have (11). Thus we obtain

$$
c(s) \leqslant \frac{1}{R^{2 \delta_{1}}} \int_{0}^{+\infty} \theta^{\delta_{1}-1} h(\theta) \mathrm{d} \theta \leqslant c \int_{0}^{+\infty} h(\theta) \mathrm{d} \theta \leqslant c,
$$


$\operatorname{since} \operatorname{supp}(h(\cdot)$ is compact. Consequently (14) and (15) entail the result:

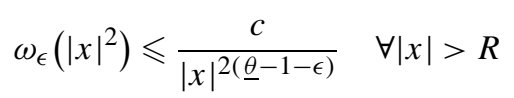

where $c$ stands for some constant independent with respect to $R$ and $\epsilon$. From the Proposition 20, (16) entail:

$$
u(x) \leqslant \frac{c}{|x|^{2(\underline{\theta}-1-\epsilon)}} \quad \forall|x|>R, \forall \epsilon>0 .
$$

This means $u(x) \leqslant c /|x|^{2(\underline{\theta}-1)}$ by monotonicity with respect to $\epsilon$.

(ii) second step. Estimate from below of $v_{\epsilon}\left(|x|^{2}\right)$.

Consider $R>0$ chosen as previously: $R>R_{\epsilon}+1$, supp $g \subset\left[0, R^{2}\right.$ [. The function $v_{\epsilon}\left(|x|^{2}\right)$ can be written for $|x|>R$

$$
\begin{aligned}
v_{\epsilon}\left(|x|^{2}\right) & =\int_{|x|^{2}}^{+\infty}\left[\int_{0}^{s} \exp \left(-\int_{\theta}^{s} \bar{q}_{\epsilon}(\sigma) \mathrm{d} \sigma\right) \frac{g(\theta)}{\theta} \mathrm{d} \theta\right] \mathrm{d} s=\int_{|x|^{2}}^{+\infty}\left[\int_{0}^{R^{2}} \exp \left(-\int_{0}^{s} \bar{q}_{\epsilon}(\sigma) \mathrm{d} \sigma\right) \frac{g(\theta)}{\theta} \mathrm{d} \theta\right] \mathrm{d} s \\
& =\int_{|x|^{2}}^{+\infty}\left[\int_{0}^{R^{2}} \exp \left(-\int_{0}^{R^{2}} \bar{q}_{\epsilon}(\sigma) \mathrm{d} \sigma\right) \exp \left(-\int_{R^{2}}^{s} \bar{q}_{\epsilon}(\sigma) \mathrm{d} \sigma\right) \frac{g(\theta)}{\theta} \mathrm{d} \theta\right] \mathrm{d} s .
\end{aligned}
$$

Using the very definition of $\bar{q}_{\epsilon}(\cdot), v_{\epsilon}\left(|x|^{2}\right)$ can be written:

$$
v_{\epsilon}\left(|x|^{2}\right)=\int_{|x|^{2}}^{+\infty} \exp \left(-\int_{R^{2}}^{s} \frac{\bar{\theta}+\epsilon}{\sigma} \mathrm{d} \sigma\right) \mathrm{d} s \int_{0}^{R^{2}} \exp \left(-\int_{\theta}^{R^{2}} \bar{q}_{\epsilon}(\sigma) \mathrm{d} \sigma\right) \frac{g(\theta)}{\theta} \mathrm{d} \theta .
$$

But we have, using (11):

$$
\begin{aligned}
& c_{\epsilon}(R)=\int_{0}^{R^{2}} \exp \left(-\int_{\theta}^{R^{2}} \bar{q}_{\epsilon}(\sigma) \mathrm{d} \sigma\right) \frac{g(\theta)}{\theta} \mathrm{d} \theta \geqslant \int_{0}^{R^{2}} \exp \left(-\int_{\theta}^{R^{2}} \frac{\delta_{2} \mathrm{~d} \sigma}{\sigma}\right) \frac{g(\theta)}{\theta} \mathrm{d} \theta, \\
& c_{\epsilon}(R) \geqslant \frac{1}{R^{2 \delta_{2}}} \int_{0}^{R^{2}} \theta^{\delta_{2}-1} g(\theta) \mathrm{d} \theta=\frac{c(R)}{R^{2 \delta_{2}}}>0
\end{aligned}
$$

where $c$ stands for some positive constant independent with respect to $R$ and $\epsilon$. In addition

$$
\int_{|x|^{2}}^{+\infty} \exp \left(-\int_{R^{2}}^{s} \frac{\bar{\theta}+\epsilon}{\sigma} \mathrm{d} \sigma\right) \mathrm{d} s=R^{2(\bar{\theta}+\epsilon)} \frac{1}{|x|^{2(\bar{\theta}-1+\epsilon)}}
$$

Thus it follows from (17), (18) and (19):

$$
v_{\epsilon}\left(|x|^{2}\right) \geqslant \frac{c(R)}{R^{2\left(\delta_{2}-\delta_{1}\right)}} \cdot \frac{1}{|x|^{2(\bar{\theta}-1+\epsilon)}} \quad \forall|x|>R, \forall \epsilon>0 .
$$

From Proposition 20, (20) entail:

$$
\frac{c(R)}{R^{2\left(\delta_{2}-\delta_{1}\right)}} \cdot \frac{1}{|x|^{2(\bar{\theta}-1+\epsilon)}} \leqslant u(x) \quad \forall|x|>R, \forall \epsilon>0 .
$$


This means

$$
\frac{c_{R}}{|x|^{2(\bar{\theta}-1)}} \leqslant u(x) \quad \forall|x|>R
$$

by monotonicity with respect to $\epsilon$. And our proof is achieved.

Now let us examine the classical interesting case: $a_{i j}(x)=\delta_{i j}$ that is to say $A$ is the Laplace operator. Then Theorem 21 says that $u(x)=c(x) /|x|^{N-2}$ for $|x|$ large enough, where $c(\cdot)$ is some function bounded from above and below, respectively, by two positive constants. We will show that the more precise is the behaviour of $\Lambda(\cdot)$, the more precise is the one of $u(\cdot)$. For this we assume hypothesis (H3). As in (7) and (9), let us define $\bar{q}_{\epsilon}(\cdot)$ and $\underline{q}_{\epsilon}(\cdot)$ in the following: for any $\epsilon>0$ there exist $R_{\epsilon}>0$ such that

$$
\forall|x| \geqslant R_{\epsilon} \quad \gamma\left(|x|^{2}\right)-\epsilon \leqslant \Lambda(x) \leqslant \gamma\left(|x|^{2}\right)+\epsilon ;
$$

let us set

$$
\begin{aligned}
& \bar{q}_{\epsilon}(t)=\frac{1}{t} \begin{cases}\gamma(t)+\epsilon, & \text { if } t \geqslant\left(R_{\epsilon}+1\right)^{2}, \\
\bar{\ell}(t), & \text { if } R_{\epsilon}^{2} \leqslant t \leqslant\left(R_{\epsilon}+1\right)^{2}, \\
\frac{\operatorname{Tr}(d)}{2 \lambda_{1}(d)}, & \text { if } 0 \leqslant t \leqslant R_{\epsilon}^{2},\end{cases} \\
& \underline{q}_{\epsilon}(t)=\frac{1}{t} \begin{cases}\gamma(t)-\epsilon, & \text { if } t \geqslant\left(R_{\epsilon}+1\right)^{2}, \\
\frac{\ell(t),}{\operatorname{Tr}(c)} & \text { if } R_{\epsilon}^{2} \leqslant t \leqslant\left(R_{\epsilon}+1\right)^{2}, \\
\frac{2 \lambda_{N}(c)}{\text { if } 0 \leqslant t \leqslant R_{\epsilon}^{2},}\end{cases}
\end{aligned}
$$

where $\bar{\ell}(\cdot), \underline{\ell}(\cdot)$, matrices $c$ and $d$ are defined as in (6), (7) and (9). We have

$$
\underline{q}_{\epsilon}\left(|x|^{2}\right) \leqslant \Lambda(x) \leqslant \bar{q}_{\epsilon}\left(|x|^{2}\right) \quad \forall x .
$$

Theorem 22. Under hypothesis (H3) there exist $R>1$ such that, for any $x \in \mathbb{R}^{N},|x| \geqslant R$, we have

$$
0 \leqslant c_{1} v\left(|x|^{2}\right) \leqslant u(x) \leqslant c_{2} v\left(|x|^{2}\right)
$$

where $c_{i}, i=1,2$, are some positive constants and

$$
v\left(|x|^{2}\right)=\int_{|x|^{2}}^{+\infty} \exp \left(-\int_{R^{2}}^{s} \frac{\gamma(\sigma)}{\sigma} \mathrm{d} \sigma\right) \mathrm{d} s .
$$

Proof. It is similar to the proof of Theorem 21. So we do not make it again.

Let us point out that we can assume an hypothesis again more satisfactory than (H3).

(H4) Suppose there exist two symmetric uniformly elliptic matrices $b(x)=\left(b_{i j}(x)\right), c(x)=\left(c_{i j}(x)\right)$, some function $\gamma: \mathbb{R}^{+} \rightarrow \mathbb{R}^{+}$and a $c^{2}$ convex function $r: \mathbb{R}^{N} \rightarrow \mathbb{R}^{+}$such that:

(i) $x^{T} c(x) x \leqslant r(x) \leqslant x^{T} b(x) x \forall x \in \mathbb{R}^{N}$,

(ii)

$$
\lim _{R \rightarrow+\infty} \sup _{|x| \geqslant R}\left|\frac{\operatorname{Tr}\left(a(x) D^{2} r(x)\right)}{2\left|\nabla_{A} r(x)\right|^{2} / r(x)}-\gamma(r(x))\right|=0,
$$

where $D^{2} r$ stands for the Hessian matrix of the function $r(\cdot)$ and $A$ is the differential operator associated to the matrix $a$. 
Using the same method as in the previous Theorems 21 and 22 we obtain the

Corollary 23. Assume (H4). Then there exist $R>0$ and two positive constants $c_{1}, c_{2}$ such that

$$
c_{1} v(x) \leqslant u(x) \leqslant c_{2} v(x) \quad \forall x,|x| \geqslant R,
$$

with

$$
v(x)=\int_{|x|^{2}}^{+\infty} \exp \left(-\int_{R^{2}}^{s} \frac{\gamma(\sigma)}{\sigma} \mathrm{d} \sigma\right) \mathrm{d} s .
$$

The proof is similar to the one of Theorem 21. But it is somewhat technical. It is left to the reader.

Remark. It is very important to consider some other behaviours of the function $\Lambda(\cdot)$ and then to state the behaviour of $u(\cdot)$. The case the matrix $\left(a_{i j}(x)\right) q$-periodic, $q=[0,1]^{N}$, is particularly interesting to study.

\section{Appendix A. An approximation result}

We have the following approximation result.

Proposition 16. Let $K(\cdot)$ be a function belonging to $L^{2}(0, T)$ and such that $K(t) / t$ belongs to $L^{1}(0, T)$. Then there exists a regular sequence of functions $K_{\eta}$ such that

(i) $K_{\eta} \rightarrow K$ strongly in $L^{2}(0, T)$ as $\eta$ goes to zero.

(ii) $K_{\eta}(\cdot) / t \rightarrow K(\cdot) / t$ strongly in $L^{1}(0, T)$ as $\eta$ goes to zero.

The proof is straightforward [11]. So we omit it.

\section{Appendix B. A regularization result}

Let us consider the solution $\omega$ of (E). In order to define $r^{\prime}(\varphi)$ and $r^{\prime \prime}(\varphi)$, let us consider the following regularized equation of (E) Section 4:

$$
-r_{\epsilon}^{\prime \prime}(t)-\frac{Q_{\epsilon}(t)}{t+\epsilon} r_{\epsilon}^{\prime}(t)=\frac{k_{\epsilon}(t)}{t+\epsilon}
$$

where $Q_{\epsilon}$ and $k_{\epsilon}$ are, respectively, a regularization of $Q$ and $k$ such that

$$
\begin{aligned}
& \lim _{\epsilon} \int_{0}^{T}\left|Q_{\epsilon}(t)-Q(t)\right|^{p} \mathrm{~d} t=0, \quad p \geqslant 2, \\
& \lim _{\epsilon} \int_{0}^{T} \frac{\left|k_{\epsilon}-k\right|}{t} \mathrm{~d} t=0, \quad \lim _{\epsilon} \int_{0}^{T}\left|k_{\epsilon}(t)-k(t)\right|^{2} \mathrm{~d} t=0 .
\end{aligned}
$$

Let us point out that $k_{\epsilon}$ exists from Appendix A, and let us recall that $Q$ and $k$ satisfy the assumptions:

$$
\int_{0}^{T} \frac{k(t)}{t} \mathrm{~d} t<+\infty, \quad 0<\delta_{1} \leqslant Q(t) \leqslant \delta_{2} .
$$


From (1), in the same way as the study of (E) we can establish that we have (see (11) Section 4):

$$
\left\{\begin{array}{l}
0 \leqslant r_{\epsilon}(t) \leqslant(T-t) \cdot \int_{0}^{T} \frac{k_{\epsilon}(s)}{s} \mathrm{~d} s \leqslant c \cdot(T-t), \\
0 \leqslant-r_{\epsilon}^{\prime}(t) \leqslant \int_{0}^{t} \frac{k_{\epsilon}(s)}{s} \mathrm{~d} s \leqslant \int_{0}^{T} \frac{k_{\epsilon}(s)}{s} \mathrm{~d} s \leqslant c,
\end{array}\right.
$$

by using (1.1). From (1) again we have:

$$
-r_{\epsilon}^{\prime \prime}(\varphi(x))-\frac{Q_{\epsilon}(\varphi(x))}{\varphi(x)+\epsilon} \cdot r_{\epsilon}^{\prime}(\varphi(x))=\frac{k_{\epsilon}(\varphi(x)}{\varphi(x)+\epsilon} \quad \forall x \in \Omega
$$

Our goal is now to pass to the limit in (3) as $\epsilon$ goes to zero. Our result is:

Proposition 17. Assume that $k(\varphi)$ belongs to $L^{2}(\Omega)$. Then we have:

$$
-A(r(\varphi)) \leqslant \frac{k(\varphi)}{\varphi}\left|\nabla_{a} \varphi\right|^{2} \quad \text { in } H^{-1}(\Omega)
$$

where $r=\lim _{\epsilon} r_{\epsilon}$, up to a subsequence, in $W^{1, \infty}(0, T)$ for the weak $*$ topology.

The proof is straightforward [11]. So we omit it.

\section{References}

[1] G. Birkhoff, Three observations on linear algebra, Universidad Nacional de Tucuman. Revista Sér. A 5 (1946) 147-151.

[2] P. Cardaliaguet, R. Tahraoui, On the strict concavity of the harmonic radius in dim $N \geqslant 3$, J. Math. Pures Appl. (9) 81 (3) (2002) $223-240$.

[3] M. Marcus, An eigenvalue inequality for the product of normal matrices, in: F.A. Ficken (Ed.), Mathematical Notes, University of Tennessee.

[4] M. Protter, H. Weinberger, Maximum Principles in Differential Equations, Prentice-Hall, 1967.

[5] R. Sperb, Maximum Principles and their Applications, Academic Press, 1981.

[6] G. Stampacchia, Le problème de Dirichlet pour les équations elliptiques du second ordre à coefficients discontinus, Ann. Inst. Fourier 15 (1965) 189-258.

[7] R. Tahraoui, Sur le Principle du Maximum des opérateurs elliptiques, C. R. Acad. Sci. Paris Sér. I 320 (1995) 1453-1458.

[8] R. Tahraoui, Générateurs infinitésimaux et propriétés géométriques pour certaines équations complètement non linéaires, Rev. Mat. Iberoamericana 11 (3) (1995).

[9] R. Tahraoui, Principe de comparaison pour opérateurs elliptiques, C. R. Acad. Sci. Paris Sér. I 322 (1996) 1053-1056.

[10] R. Tahraoui, Maximum Principle for elliptic operators and applications, Ann. Inst. H. Poinaré Analyse Non Linéaire 19 (6) (2002) 815870.

[11] R. Tahraoui, Comparison Principle for second order elliptic operators and applications, Ceremade UMR 7534, Université Paris-Dauphine, $\mathrm{N}^{\circ}$ 0344, 3 Décembre 2003. 